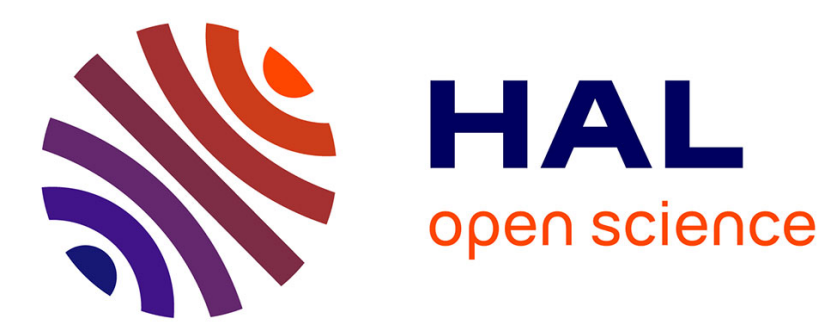

\title{
Analysis of the modal energy distribution of an excited vibrating panel coupled with a heavy fluid cavity by a dual modal formulation
}

\author{
Laurent Maxit
}

\section{- To cite this version:}

Laurent Maxit. Analysis of the modal energy distribution of an excited vibrating panel coupled with a heavy fluid cavity by a dual modal formulation. Journal of Sound and Vibration, 2013, 332, pp.6703-6724. 10.1016/j.jsv.2013.07.020 . hal-00879258

\section{HAL Id: hal-00879258 https://hal.science/hal-00879258}

Submitted on 2 Nov 2013

HAL is a multi-disciplinary open access archive for the deposit and dissemination of scientific research documents, whether they are published or not. The documents may come from teaching and research institutions in France or abroad, or from public or private research centers.
L'archive ouverte pluridisciplinaire HAL, est destinée au dépôt et à la diffusion de documents scientifiques de niveau recherche, publiés ou non, émanant des établissements d'enseignement et de recherche français ou étrangers, des laboratoires publics ou privés. 


\title{
Analysis of the modal energy distribution of an excited vibrating panel coupled with a heavy fluid cavity by a dual modal formulation
}

\author{
Laurent MAXIT \\ INSA Lyon, Laboratoire Vibrations-Acoustique (LVA), \\ 25 bis, av. Jean Capelle, 69621 Villeurbanne, France \\ laurent.maxit@insa-lyon.fr \\ Tel. +33472438082 \\ Fax. +33472438712
}

\section{ABSTRACT}

This paper describes the modal interaction between a panel and a heavy fluid cavity when the panel is excited by a broad band force in a given frequency band. The Dual Modal Formulation (DMF) allows describing the fluid-structure coupling using the modes of each uncoupled subsystem. After having studied the convergence of the modal series on a test case, we estimate the modal energies and the total energy of each subsystem. An analysis of modal energy distribution is performed. It allows us to study the validity of SEA assumptions for this case. Added mass and added stiffness effects of the fluid are observed. These effects are related to the non resonant acoustic modes below and above the frequency band of excitation. Moreover, the role of the spatial coupling of the resonant cavity modes with the non resonant structure modes is also highlighted. As a result, the energy transmitted between the structure and the heavy fluid cavity generally cannot be deduced from the SEA relation established for a light fluid cavity.

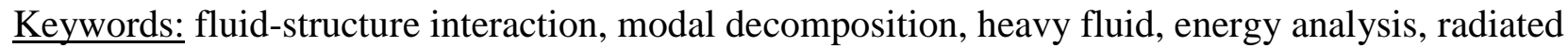
noise 


\section{INTRODUCTION}

The fluid-structure interaction between a flexible structure and an air cavity has been studied frequently by many authors [1-5], in particular in the framework of Statistical Energy Analysis (SEA) models [5-9].

The Dual Modal Formulation (DMF) [10] of the structure-cavity system consists in considering the in-vacuo modes of the structure and the rigid wall modes of the cavity. The modal equation of motion shows that each mode of one subsystem is coupled with the modes of the other subsystem. There is no direct coupling between the modes of the same subsystem, as shown in Fig. 1.

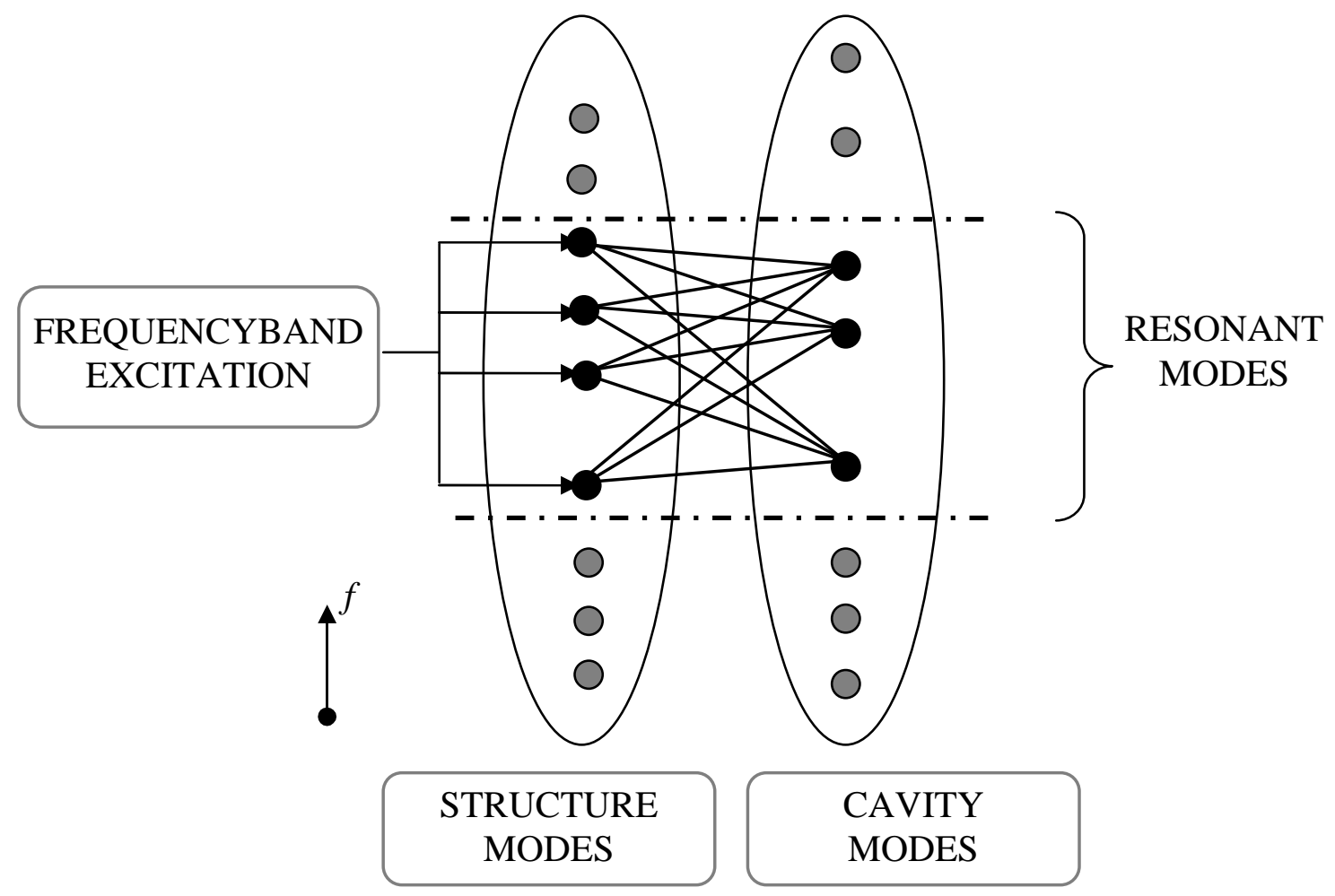

Fig. 1. Illustration of the modal coupling for an air cavity-structure excited by a broad band force.

As air is a light fluid (i.e. small bulk modulus), there is considerable impedance discontinuity between the structure and the cavity. The global modes of the system are similar in shape and frequencies to the subsystem modes. The coupling between the structure and the air cavity is "weak", which is an assumption of the classical SEA model [11, 12]. Indeed, under this assumption, the SEA assumes that the interaction between the structure and the cavity for a broad band excitation can be sufficiently described by the interactions between the resonant modes of each subsystem, as illustrated in Fig. 1. Then, the energy sharing between the two subsystems in a given frequency band can be estimated from the energy sharing by its resonant modes. Moreover, in the classical SEA formulation, the power flow between two resonant modes is estimated from a relation established for two coupled oscillators excited by uncorrelated white noise. 
Considering these two assumptions (i.e. resonant mode domination, power flow estimate by pairs of modes) and assuming modal energy equi-partition, the SEA model indicates that the power flows from the structure to the cavity $E_{s \rightarrow c}$ are proportional to the difference of the mean of the modal energy of the structure $\bar{e}_{s}$ and the cavity $\bar{e}_{c}$ :

$$
E_{s \rightarrow c}=\omega_{c} N_{s} \eta_{s-c}\left(\bar{e}_{s}-\bar{e}_{c}\right),
$$

where $\omega_{c}$ is the central angular frequency of the frequency band considered, $N_{s}$ is the number of resonant modes of the structure and $\eta_{s-c}$ is the Coupling Loss Factor (CLF) between the structure and the cavity.

The Coupling Loss Factor expresses the coupling strength between the two subsystems. Different methods exist for estimating this parameter for a structure-cavity system. It can be approximated from the radiation efficiency of a baffle plate. In this case, it is independent from the damping loss factor of the structure. Another method consists in summing the modal coupling factors of pairs of resonant modes [7]. Fahy used this technique to estimate the response of a containing structure to broad-band sound in the enclosed air [7,8]. The response was usually found to be the same as that of a diffuse acoustic field in the external fluid above a "lower limiting frequency" determined by the geometry and the mechanical properties of the system. He also showed that few pairs of modes contribute significantly to the energy sharing between the structure and the cavity due to the spatial and frequency coincidence phenomena. Comparisons with experimental measurements were performed to validate his developments. The cases considered by Fahy were academic: a rigid rectangular box with a simply-supported flexible wall and a closed cylindrical shell with the enclosed air. More recently, Torato and Guyader [13] have extended this technique to complex systems by extracting the modal information with Finite Element models of each subsystem (i.e. structure, cavity). Culla and Sestieri [14] studied the validity of the SEA relation (1) for a structure-cavity system. The results of a deterministic model were compared with the SEA results for a rigidly bounded air cavity coupled with a simply supported plate excited by mechanical forces. The authors found that the results agree well if the modal overlap factors of the plate and the cavity are greater than one. This condition is one of the basic assumptions of the SEA method [6]. Indeed, the modal energy equipartition assumption is not respected for low modal overlap as highlighted in [15]. Recently, Lei et al. [9] proposed an improved SEA model for predicting the structural response and noise reduction of acoustical enclosures. The model presented included the resonant and the non resonant responses of panels. Good agreement between the prediction and measured results was observed even when few modes were resonant.

All the works mentioned above concern a flexible structure coupled with an air cavity. In this paper, we are interested in studying the influence of a heavy fluid in the cavity instead of a light fluid like air. This case has applications in the nuclear and the submarine industries. For example, for the design of the Sonar dome of a submarine, it could be relevant to estimate the noise level in the Sonar cavity when the dome is excited by pressure fluctuations in the turbulent boundary layer [16]. The dome may be represented by a thin flexible structure, the Sonar cavity by an enclosure filled with water and the sea water by a "semi infinite" fluid medium. In the present paper, the effect of the sea water on the dome will not be investigated. Different studies have already highlighted the added mass effect of a "semi-infinite" fluid environment on the vibrating structure for frequencies below the critical frequency [17]. In the present paper, we will focus our attention on the modal interaction between a thin structure and a closed cavity filled with a heavy fluid. In the SEA formulation, the two assumptions described above (i.e. resonant mode domination and power flow estimated by pairs of modes) are generally valid for a light fluid. We will study their validity in the case of 
heavy fluid in order to apply an SEA method or to develop an adapted SEA method for this case in the future. Liu et al. [18] were interested in modelling liquid-structure interactions within the framework of statistical energy analysis. However, in their work, they assume that the SEA relation (1) is valid for cavities filled with water, though without providing evidence. In another context, David and Menelle [19, 20] highlighted the added mass and added stiffness effects of a heavy fluid-filled cavity to a vibrating structure. They used a $(\mathrm{u}, \phi)$ formulation of the problem including the static displacement potential and the static pressure. Comparisons of their results with experimental measurements showed very good agreement. The test case considered in [20] cannot be considered in the present study because the plate and the cavity have very few modes up to $5 \mathrm{kHz}$. For the purposes of validation, however, we will present a comparison of the DMF results with their results.

Our analysis will be based on a test case composed of a rectangular flat plate coupled with a parallelepiped water-filled cavity. This case has been chosen for its simplicity. The modal information of each subsystem can be calculated analytically. This enables us to facilitate studying the convergence of the modal decomposition for the present case. Finite element models could be used to extract the modal information for more complex cases [13], with increased computing effort. This is not however the objective of this study.

The present paper is organised as follow:

- In section II, we will recall the results of the Dual Modal Formulation which enables us to represent the fluid-structure interaction of the thin structure - cavity system from the interaction of the uncoupled modes of each subsystem (structure and cavity). We will establish the relations between the modal amplitudes, the modal energies and the total energy of each subsystem. Finally, we describe the calculation process used for evaluating the energy for broad band excitation;

- Section III presents the plate-cavity test case and a study of the convergence of the modal series when the cavity is filled with a heavy fluid (water). A reference result is obtained by performing Finite Element calculations with a direct resolution. The comparisons highlight the influence of the non-resonant modes of the plate and the cavity. The DMF results are also compared with the results obtained by David and Menelle [20] in appendix A for the purposes of validation;

- Section IV deals with the analysis of the modal energy distributions between the plate and the cavity considering the non-resonant modes. The effects of these non-resonant modes are discussed on the basis of the modal equations. The well-known fluid added mass effect is expressed in terms of non-resonant contributions and an added stiffness effect and the role of the first non-resonant plate modes are highlighted. An approximate model of the modal interaction is finally deduced.

- In section V, we will compare, for different damping and different frequencies, the energy responses obtained with the classical SEA with those obtained with the DMF (basic or approximate models). The different effects described in section IV are then studied as a function of the frequency and the damping of the plate.

The paper will be concluded by perspectives on the present developments.

\section{DUAL MODAL FORMULATION (DMF)}

DMF can be used for calculating the force response of two coupled subsystems from the knowledge of the uncoupled subsystem modes. This modal formulation has long been known for describing the dynamic behaviour of a flexible structure coupled with a close acoustic 
domain. A Green formulation [10, 21] or a variational formulation of the fluid-structure problem [22] may be used for obtaining the modal equation of motion. Otherwise, DMF has been extended to the general case of the coupling of two elastic continuous mechanical systems [23]. The modal interaction diagram obtained with this formulation is similar to the diagram assumed in the classical SEA formulation [6] (see Fig. 1). This approach is therefore well adapted for investigating the effect of a heavy fluid cavity on the SEA method.

\subsection{Calculation of the forced response}

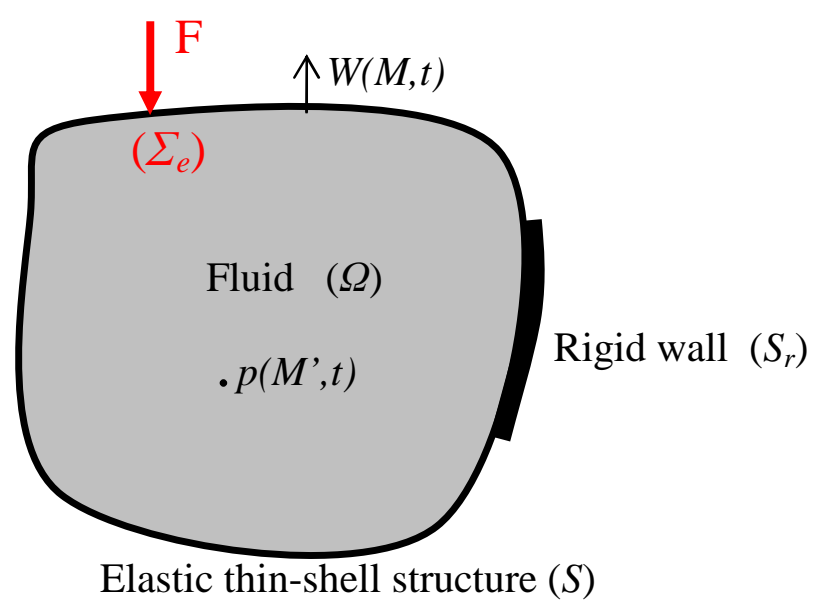

Fig. 2. Vibrating structure coupled with a heavy fluid cavity and excited by a mechanical force.

Let us consider the internal vibro-acoustic problem presented in Fig 2. The vibrating structure is coupled with a rigid-walled acoustic cavity of volume $\Omega . S$ is the fluid-structure coupling interface and $S_{r}$ is the rigid wall surface. The structure is assumed to be thin, elastic and homogeneous. $h, \rho, \eta_{1}$ are, respectively, the thickness, the mass density and the damping loss factor of the structure. A random point force applied at point $\Sigma_{e}$ excites this structure. The Auto-Spectrum Density (ASD) of the force, $S_{F F}$ is assumed to be constant (i.e. white spectrum) in the frequency band with a central frequency $\omega_{c}$ and a width $\Delta \omega$. The internal fluid is modelled with the Helmholtz equation. $c_{0}, \rho_{0}, \eta_{2}$ are the celerity, the mass density and the damping loss factor, respectively, of the fluid.

\subsubsection{Formulation}

Let us consider DMF which is based on modal decompositions with the modes of each uncoupled subsystem [10, 23]. The thin structure is described by a displacement field (i.e. normal displacement) and its uncoupled-free modes (i.e. in-vacuo structure modes) whereas the cavity is described by a stress field (i.e. acoustic pressure) and its uncoupled-blocked modes (i.e. rigid wall cavity modes). These uncoupled modes can be easily calculated analytically for academic cases $([7,8])$ or numerically with Finite Element models for complex cases ([13]). 
The modal expansions of the normal displacements, $W$ and the acoustic pressure, $p$ may be written:

$$
\begin{aligned}
& W(M, t)=\sum_{p=1}^{\infty} \chi_{p}(t) \tilde{W}_{p}(M), \\
& p\left(M^{\prime}, t\right)=\sum_{q=1}^{\infty} \xi_{q}(t) \tilde{p}_{q}\left(M^{\prime}\right),
\end{aligned}
$$

where: $-\tilde{W}_{p}$ and $\chi_{p}$, are, respectively, the displacement shape and the amplitude of the $p^{\text {th }}$ mode of the structure;

- $\tilde{p}_{q}$ and $\xi_{q}$, are, respectively, the pressure shape and the amplitude of the $q^{\text {th }}$ mode of the cavity.

Hereafter, the space and time dependencies of the notation will be deleted but they are still considered. DMF consists in introducing these expansions (2-3) in a weak formulation of the vibro-acoustic problem considered and using the orthogonality properties of the uncoupled modes. For more details on this formulation, the reader can study Ref. [10, 21-23].

Finally, with the change of variable,

$$
\xi_{q}=\zeta_{q}^{\prime}
$$

the following modal equation system is obtained

$$
\left\{\begin{array}{l}
M_{p}\left(\chi_{p}^{\prime \prime}+\omega_{p} \eta_{1} \chi_{p}^{\prime}+\omega_{p}^{2} \chi_{p}\right)-\sum_{q=1}^{\infty} W_{p q} \zeta_{q}^{\prime}=F_{p}, \forall p \in[1,+\infty[ \\
K_{q}\left(\zeta_{q}^{\prime \prime}+\omega_{q} \eta_{2} \zeta_{q}^{\prime}+\omega_{q}^{2} \zeta_{q}\right)+\sum_{p=1}^{\infty} W_{p q} \chi_{p}^{\prime}=0, \forall q \in[1,+\infty[
\end{array}\right.
$$

where:

- $M_{p}, F_{p}$ are the generalised mass and the generalised force of mode $p$ :

$$
M_{p}=\rho \int_{S} h \tilde{W}_{p}^{2} d S, \text { and } F_{p}=F_{0} \tilde{W}_{p}\left(\Sigma_{e}\right),
$$

with $F_{0}$, the amplitude of the external force;

- $K_{q}$ is the generalised stiffness of mode $q$ :

$$
K_{q}=\frac{1}{\rho_{0} c_{0}^{2}} \int_{\Omega} \tilde{p}_{q}^{2} d V
$$

- $\omega_{p}$ and $\omega_{q}$ are the modal angular frequencies of mode $p$ and $q$, respectively,

- $W_{p q}$ is the work of interaction between mode $p$ and $q$ :

$$
W_{p q}=\int_{S} \tilde{W}_{p} \tilde{p}_{q} d S
$$

It can be observed that the equation system (5) can be interpreted as the coupling between a set of oscillators associated with the thin structure with another set of oscillators associated with the cavity. The coupling elements called gyroscopic elements are related to the oscillator's velocities without dissipation of energy (due to the opposite signs in Eq. 5). On the other hand, there is no direct coupling between the oscillators of the same subsystem. This configuration of mode coupling is exactly what is assumed by SEA ([6]).

In theory, the modal decompositions (2) and (3) need an infinite number of terms. In practice, SEA considers only the resonant modes contained in the frequency band of excitation and assumes that these modes are sufficient for describing the vibro-acoustic behaviour of the 
system considered. This assumption will be studied in sections 3 and 4 of this paper. In the present developments, we consider that the structure may be described by $P$ modes and the cavity by $Q$ modes without describing how these modes are selected.

\subsubsection{Resolution}

The time-averaged energy of each subsystem will be estimated in section 2.3 from frequency transfer functions. To evaluate these transfer functions in Fourier domain, assuming that the excitation force $F(t)=F_{0} \times e^{j \omega t}$ and the solution $x(t)=\bar{X} \times e^{j \omega t}$, the system of linear equations (5) can be expressed in a matrix form as:

with the matrices:

$$
\left[\begin{array}{cc}
Z_{11} & -j \omega W_{12} \\
+j \omega W_{12}{ }^{T} & Z_{22}
\end{array}\right]\left[\begin{array}{c}
\mathrm{X}_{1} \\
Y_{2}
\end{array}\right]=\left[\begin{array}{c}
F_{1} \\
0
\end{array}\right]
$$

$$
\begin{gathered}
\mathrm{X}_{1}=\left[\begin{array}{c}
\vdots \\
\bar{\chi}_{p} \\
\vdots
\end{array}\right]_{P \times 1}, Y_{2}=\left[\begin{array}{c}
\vdots \\
\bar{\zeta} \\
\vdots \\
\vdots
\end{array}\right]_{Q \times 1}, F_{1}=\left[\begin{array}{c}
\vdots \\
\bar{F}_{p} \\
\vdots
\end{array}\right]_{P \times 1}, \\
Z_{11}=\operatorname{diag}\left[M_{p}\left(-\omega^{2}+j \omega \omega_{p} \eta_{1}+\omega_{p}{ }^{2}\right)\right]_{P \times P}, \\
Z_{22}=\operatorname{diag}\left[K_{q}\left(-\omega^{2}+j \omega \omega_{q} \eta_{2}+\omega_{q}^{2}\right)\right]_{Q \times Q}, \\
W_{12}=\left\lfloor W_{p q}\right\rfloor_{P \times Q} .
\end{gathered}
$$

The solution of this system is given by:

$$
\begin{gathered}
\mathrm{X}_{1}=\left(Z_{11}-\omega^{2} W_{12} Z_{22}{ }^{-1} W_{12}{ }^{T}\right)^{-1} F_{1}, \text { and }, \\
Y_{2}=-j \omega Z_{22}{ }^{-1} W_{12}{ }^{T} X_{1} .
\end{gathered}
$$

Since matrix $Z_{22}$ is diagonal, its inverse is easily calculated. Eq. (14) requires inverting a square matrix $P \times P$ (i.e. matrix of dimensions equal to the structure mode number). The time necessary for this calculation does not depend on the mode number $Q$ considered for the cavity. It is then possible to consider a large mode number for the cavity provided that these modes can be calculated.

\subsection{Relations between modal amplitudes, modal energies, and subsystem energies}

The modal amplitude calculated with relations (14) and (15) can be used to estimate the modal energy distribution and the total energy of each subsystem. We establish here the different relations for the cavity. Similar ones can be obtained for the panel.

Let us consider the instantaneous strain energy of the cavity given by

$$
E_{2}^{S}(t)=\frac{1}{2 \rho_{0} c_{0}^{2}} \int_{\Omega} p(M, t)^{2} d V .
$$

Introducing the modal expansion of the acoustic pressure (3) and taking the mode's orthogonality into account, we obtain 


$$
E_{2}^{S}(t)=\sum_{q=1}^{Q} E_{q}^{K}(t)
$$

where $E_{q}^{K}(t)=\frac{1}{2} K_{q} \zeta_{q}^{\prime}(t)^{2}$ represents the kinetic energy of the $q^{t h}$ oscillator (of mass $K_{q}$ ).

The strain energy of the cavity is therefore related to the modal kinetic energy. This result, which may appear surprising, is due to the fact that the displacement (i.e. amplitude) of the oscillator corresponds to the modal pressure.

Similarly, the kinetic energy of the cavity is related to the modal strain energy:

$$
E_{2}^{K}(t)=\sum_{q=1}^{Q} E_{q}^{S}(t)
$$

with $E_{q}^{S}(t)=\frac{1}{2} M_{q} \zeta_{q}(t)^{2}$

By adding Eqs. (17) and (18), we deduce that the total energy of the cavity is equal to the sum of its modal energies (defined as the sum of modal kinetic energy and the modal strain energy).

Then, if the considered modes are able to represent the vibro-acoustic behaviour of the panelcavity system, the total energy of a given subsystem can be obtained by summing its modal energies. This relies on the orthogonality of the subsystem modes, with no additional assumptions.

\subsection{Calculation process for estimating the time-averaged energies}

To reach agreement with the SEA method, it is necessary to evaluate the time-averaged energies of each subsystem considering the white noise force in the frequency band $\Delta \omega$.

In what follows, the time averaged energy $E(t)$ is noted $\langle E\rangle_{t}$ and defined by

$$
<E>_{t}=\lim _{T \rightarrow+\infty} \frac{1}{2 T} \int_{-T}^{+T} E(t) d t
$$

Applying the frequency decomposition adapted to stationary random processes [24], the timeaveraged energies for the mode $p$ of the structure can be estimated by,

$$
<E_{p}^{K}>_{t}=\frac{1}{2} \int_{\Delta \omega} M_{p} \omega^{2} S_{\chi_{p} \chi_{p}}(\omega) d \omega,<E_{p}^{S}>_{t}=\frac{1}{2} \int_{\Delta \omega} M_{p} \omega_{p}{ }^{2} S_{\chi_{p} \chi_{p}}(\omega) d \omega,
$$

and for mode $q$ of the cavity by,

$$
<E_{q}^{K}>_{t}=\frac{1}{2} \int_{\Delta \omega} K_{q} \omega^{2} S_{\zeta_{q} \zeta_{q}}(\omega) d \omega,<E_{q}^{S}>_{t}=\frac{1}{2} \int_{\Delta \omega} K_{q} \omega_{q}^{2} S_{\zeta_{q} \zeta_{q}}(\omega) d \omega .
$$

$S_{\chi_{p} \chi_{p}}(\omega), S_{\zeta_{q} \zeta_{q}}(\omega)$ are the Auto-Spectrum Densities (ASD) of the modal amplitudes, $\chi_{p}$ and $\zeta_{q}$, respectively. These quantities can be calculated using the ASD of the external force, $S_{F F}(\omega)$ and the transfer functions $H_{F \chi_{p}}(\omega)$ and $H_{F \zeta_{q}}(\omega)$ using

$$
S_{\chi_{p} \chi_{p}}(\omega)=S_{F F}\left|H_{F \chi_{p}}(\omega)\right|^{2}, S_{\zeta_{q} \zeta_{q}}(\omega)=S_{F F}\left|H_{F \zeta_{q}}(\omega)\right|^{2} \text {. }
$$


$H_{F \chi_{p}}(\omega)$ (resp. $H_{F \chi_{p}}(\omega)$ ) corresponds to the ratio of the modal amplitude $\chi_{p}$ (resp. $\zeta_{q}$ ) over the force amplitude for an harmonic excitation at the angular frequency $\omega$. These quantities can be calculated using relations (14) and (15) of section 2.1.2.

The integrals of $(20,21)$ cannot be solved analytically. In what follows, they will be estimated numerically by using the rectangular rule with a frequency step $\delta \omega$ defined in accordance with the smallest damping bandwidths of the two subsystems $\left(\delta \omega=\omega_{c} \min \left[\eta_{1}, \eta_{2}\right] / 6\right)$. Thus we can estimate the time-averaged energy of each mode and deduce the total energy of each subsystem by summation to obtain the quantities used by SEA.

\section{NUMERICAL VALIDATION}

\subsection{Presentation of the test case}

As shown in Fig. 3, we consider a system composed of a rectangular simply-supported plate coupled with a parallelepiped cavity. The geometric and mechanical parameters are chosen to be relevant with a naval application concerning the Sonar dome-cavity system of a submarine. The elastic homogeneous plate $2 \mathrm{~m}$ long, $1.8 \mathrm{~m}$ wide and $8 \mathrm{~mm}$ thick is made of steel (mass density $\rho=7800 \mathrm{~kg} / \mathrm{m}^{3}$, Young modulus $E=2.10^{11} \mathrm{~Pa}, \eta_{l}=0.01$ ). The cavity is filled with water (mass density $\rho_{0}=1000 \mathrm{~kg} / \mathrm{m}^{3}$, celerity $c_{0}=1500 \mathrm{~m} / \mathrm{s}$, damping loss factor $\eta_{2}=0.01$ ) and has a depth of $1.4 \mathrm{~m}$. The behaviour of the plate may be described by the Kirchhoff equation whereas the Helmholtz equation may be considered for the fluid domain. The excitation and receiving points are defined in the coordinate system $(O, x, y, z)$ shown in Fig. 3. The plate is assumed excited by a point force on the plate at $(0.3 \mathrm{~m}, 0.5 \mathrm{~m}, 1.4 \mathrm{~m})$. For the purpose of validation, this excitation is assumed here to be harmonic at the angular frequency $\omega$.

\subsection{FEM simulation}

A reference result is obtained by using the Finite Element (FE) method. The FE meshing shown in Fig. 3 was defined to authorise calculations up to $800 \mathrm{~Hz}$. The frequency range [1 $\mathrm{Hz}-800 \mathrm{~Hz}$ ] is well below the critical frequency of the $8 \mathrm{~mm}$ thick plate immerged in water (around $28 \mathrm{kHz}$ ). For these frequencies, the flexural wavelength of the plate is much smaller than the acoustic wavelength of the cavity. The criterion for the element size (i.e. 6 elements per wavelength) was based on the flexural wavelength, both for the plate meshing and the cavity meshing, in order to deal with coincident meshing. This makes it possible to provide a fine description of the radiated pressure field close to the plate (due to evanescent waves). 


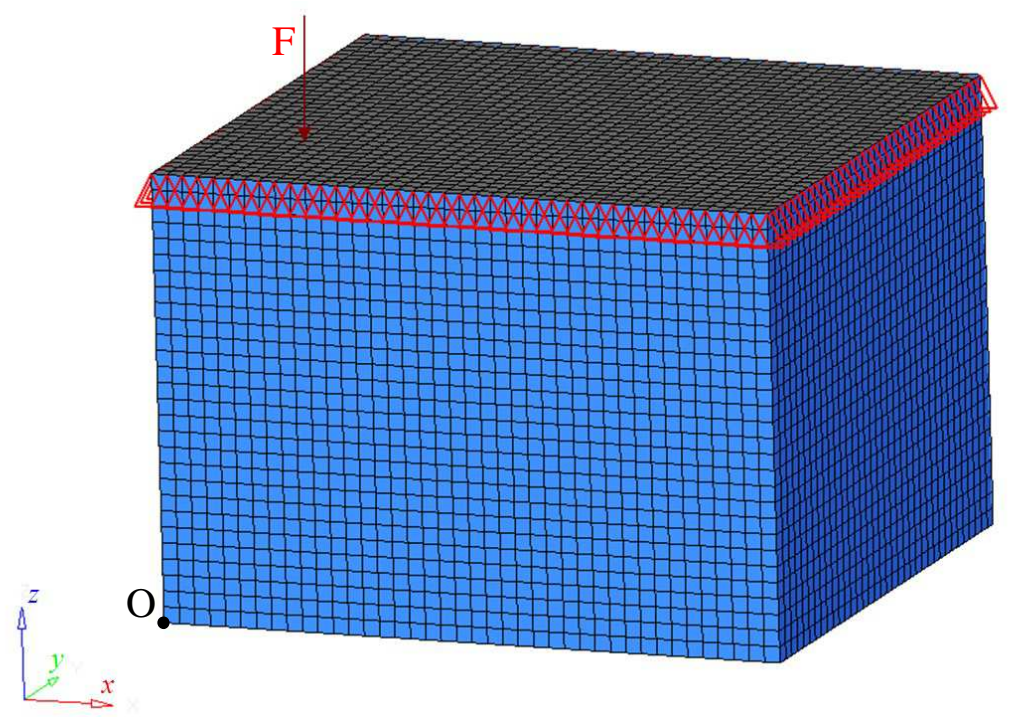

Fig. 3. Finite Element meshing of the plate-cavity system. 45510 nodes, 40320 3D-elements, 1440 2D-elements.

The FE discretisation of the motion equation of the fluid-structure problem for a harmonic excitation on the plate may be written as:

$$
\left[\begin{array}{cc}
K_{S} & -A \\
0 & K_{F}
\end{array}\right]\left\{\begin{array}{l}
U \\
P
\end{array}\right\}-\omega^{2}\left[\begin{array}{cc}
M_{S} & 0 \\
A^{T} & M_{F}
\end{array}\right]\left\{\begin{array}{l}
U \\
P
\end{array}\right\}=\left\{\begin{array}{l}
F \\
0
\end{array}\right\},
$$

where: - $U$ and $P$ represent the nodal displacements of the structure and the nodal pressure in the cavity, respectively;

- $F$ are the nodal forces applied on the structure;

- $M_{S}$ and $K_{S}$ are the mass and stiffness matrices, respectively, of the structure;

- $M_{F}$ and $K_{F}$ are the mass and stiffness matrices, respectively, of the cavity;

- $A$ is the fluid-structure interaction matrix, and subscript $\mathrm{T}$ refers to the transposed matrix.

The damping effect is introduced by considering a complex Young modulus for the structure and a complex celerity in the fluid. $K_{S}$ and $K_{F}$ are therefore complex matrices. Two Finite Element codes were used to generate the matrix system (23): MSC/NASTRAN was used for calculating $K_{S}, M_{F}, K_{F}$ and $A$, whereas SDtools [25] gave the "consistent mass" matrix of the structure, $M_{S}$. The use of SDtools was necessary because the "diagonally lumped mass" matrix calculated by MSC/NASTRAN did not give satisfactory results. Indeed, in this case, the mass matrix was approximated by distributing the element mass in the nodal translational directions. No inertial terms were considered for the nodal rotational direction. The results converged slowly as a function of mesh fineness (as observed in [26]). On the contrary, the "consistent mass" matrix was defined consistent with the shape functions and the variational formulation of the problem. It preserved angular momentum (see Chap. 32 of [27] for details on consistent mass matrices). We observed that these rotational terms played a significant role in the interaction of the structure with the heavy fluid and cannot be neglected.

The different matrices were imported in MATLAB and the system (23) was solved for each frequency with the inverse matrix method. These calculations were performed in the band [1 $\mathrm{Hz}-800 \mathrm{~Hz}$ ] with a frequency resolution of $1 \mathrm{~Hz}$. We underline that these FEM calculations did not use modal expansions and are quite appropriate for studying the convergence of the modal series of the DMF calculation. 


\subsection{Comparison between DMF and FEM results}

DMF calculations were performed by considering the subsystem modes contained in the enlarged frequency band $[0 \mathrm{~Hz}-1200 \mathrm{~Hz}] .156$ plate modes and 22 cavity modes were therefore considered. The modal information and the modal interaction work of the present case are given in appendix B.

To ensure consistency with the hysteretic damping model of the FE simulation, it was necessary to consider a modal hysteretic damping instead of a modal viscous damping in the DMF equation (9). Then, for these validation calculations, we considered the following impedance matrices (instead of $(11,12)$ ):

$$
Z_{11}=\operatorname{diag}\left[M_{p}\left(-\omega^{2}+\omega_{p}^{2}\left(1+j \eta_{2}\right)\right)\right]_{P \times P}, Z_{22}=\operatorname{diag}\left[K_{q}\left(-\omega^{2}+\omega_{q}^{2}\left(1+j \eta_{2}\right)\right)\right]_{Q \times Q} .
$$

(a)

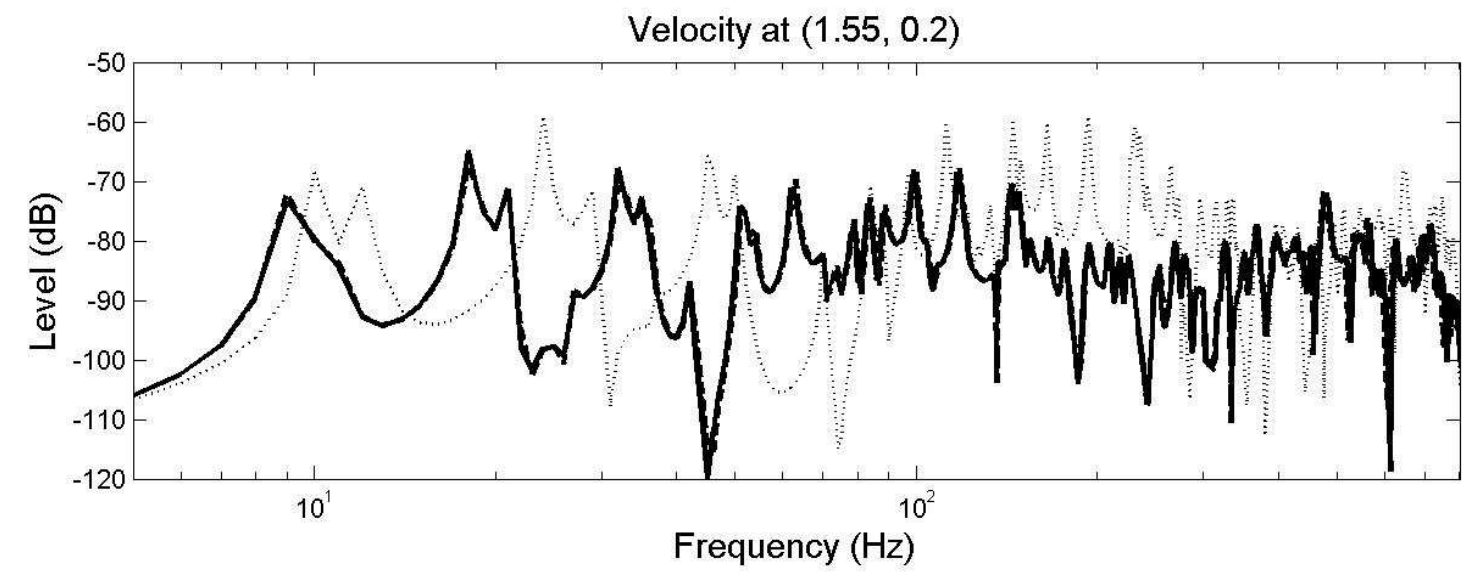

(b)

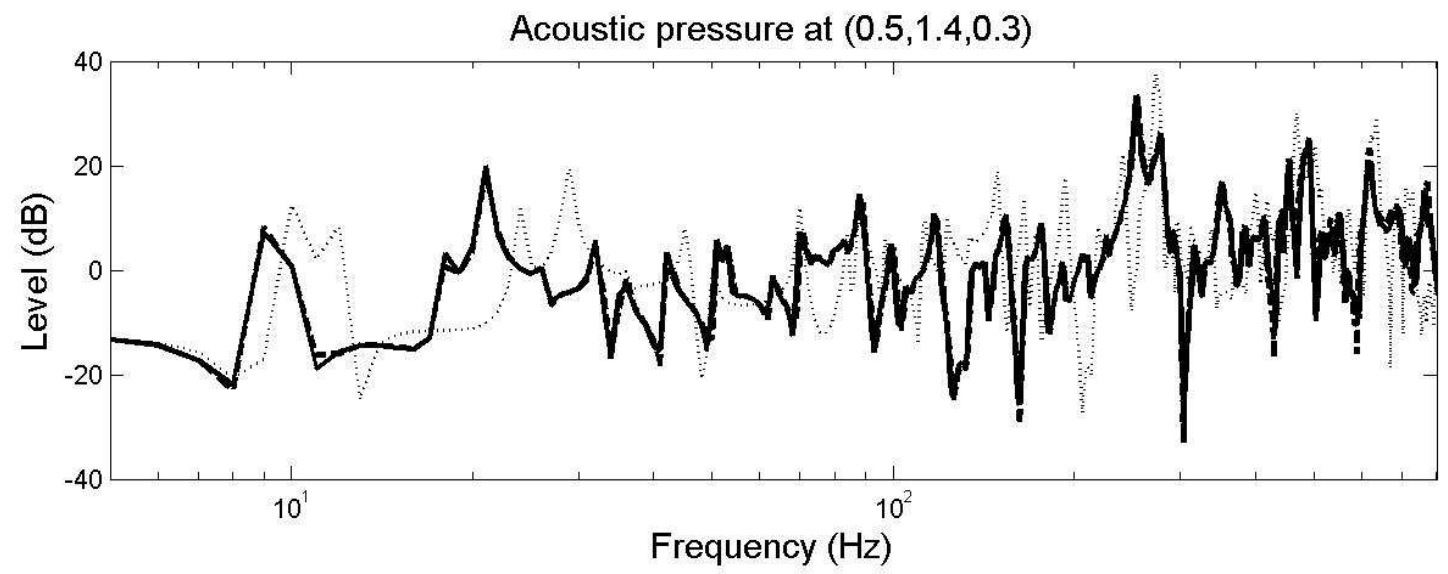

Fig. 4. Comparison between 3 calculations: FEM results (full line); DMF results taking the modes below $1200 \mathrm{~Hz}$ (dotted line), DMF results taking into account the acoustic modes spatially coincident with the plate modes (dashed line). (a), velocity response on the plate at point $(1.55 \mathrm{~m}, 0.2 \mathrm{~m}, 1.4 \mathrm{~m})$; (b), pressure response in the cavity at point $(0.5 \mathrm{~m}, 1.4 \mathrm{~m}, 0.3$ 
In Fig. 4 we compare the DMF (dotted line) and FE results (full line) for two receiving points: one on the plate and the other one inside the cavity. Large discrepancies can be seen, indicating that the modes considered and the DMF equations cannot describe the behaviour of the present system correctly. Contrary to a light fluid, the resonant modes of each subsystem are not accurate here for describing the behaviour of the coupled subsystem. This is due to significant contributions of the non-resonant acoustic modes which coincide spatially with the resonant structure modes. As we will see later, spatially coincident acoustic modes create an added mass effect to the structure which is not taken into account in the DMF calculation. These spatial coincidences intervene in the DMF equations through the intermodal work $W_{p q}$ (see Eq. (B.7)). In order to highlight the strongest spatial couplings, in Fig. 5 we show the couples of modes which present significant values for $\left|W_{p q}\right|$. The intermodal work is defined here as significant in comparison with the highest value, $\bar{W}=\max _{\substack{p \in P \\ q \in Q}}\left|W_{p q}\right|$. If $\left|W_{p q}\right|$ is greater than half of $\bar{W}$, the intermodal work is significant. For the sake of clarity, only the cavity modes with a null modal order in the direction perpendicular to the plate (i.e. $q_{z}=0$ ) are considered in this figure.

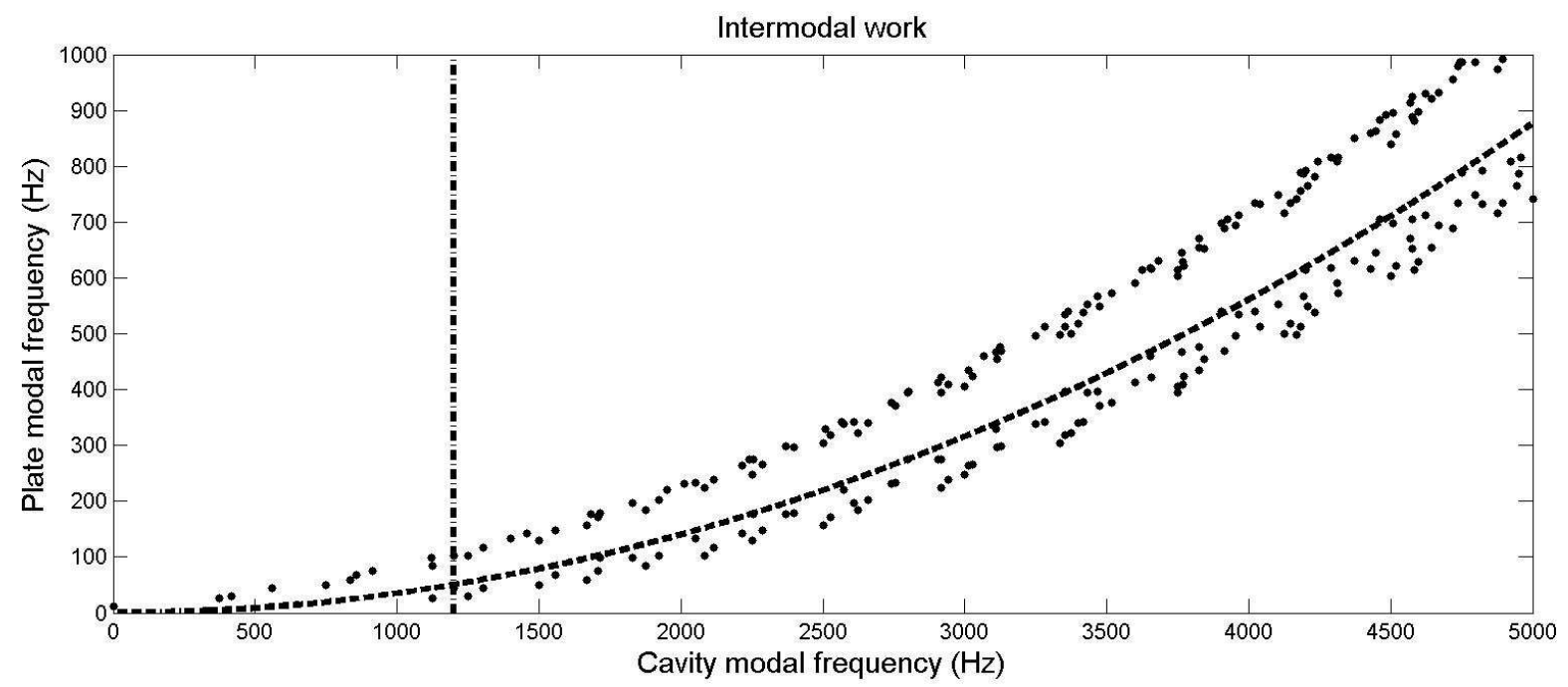

Fig. 5. Positions of the significant intermodal works $W_{p q}$ as a function of the frequencies of plate and cavity modes $\left(\omega_{q}, \omega_{p}\right)$. Only cavity modes with a null modal order in the $z$ direction are considered (i.e. $q_{z}=0$ ). Dashed line, frequencies given by Eq. (26).

We observe that the plate modes with a frequency higher than $100 \mathrm{~Hz}$ exhibit significant intermodal work with non resonant cavity modes (i.e. modes with a frequency higher than 800 $\mathrm{Hz}$ ). These plate modes coincide spatially with non-resonant cavity modes. Equation (B.7) of the intermodal work $W_{p q}$ for a given couple of plate modal orders $\left(p_{x}, p_{y}\right)$, with a variation of the cavity modal orders $\left(q_{x}, q_{y}\right)$, shows that $W_{p q}$ is maximum for $q_{x}=p_{x} \pm 1$, and $q_{y}=p_{y} \pm 1$, simultaneously. On the other hand, the magnitude of $\left|W_{p q}\right|$ is independent of the modal order in the direction perpendicular to the plate, $q_{z}$. It is not possible to establish a relation between the frequencies of the spatially coincident modes. 
However, if we consider the modal conditions $q_{x}=p_{x}, q_{y}=p_{y}$, we obtain a relation between the modal frequencies of the plate $\tilde{f}_{p}$ and the cavity $\tilde{f}_{q}$ :

$$
\tilde{f}_{q}\left(q_{z}\right)=\frac{c_{0}}{2 \pi} \sqrt{2 \pi \tilde{f}_{p} \sqrt{\frac{\rho h}{D}}+\left(\frac{\pi q_{z}}{L_{z}}\right)^{2}} .
$$

This relation gives us a first approximation of the frequency of the cavity modes which coincide spatially with a given plate mode. This is illustrated in Fig. 5 where a dashed curve corresponding to (25) with $q_{z}=0$ is plotted. The significant intermodal works can be seen on either side of this curve. For $\tilde{f}_{p}=800 \mathrm{~Hz}$, we obtain $\tilde{f}_{q}(0)=4775 \mathrm{~Hz}$. This indicates that for the validation case, the "last" resonant plate mode is spatially coincident with cavity modes $q_{z}=0$ of frequency around $4700 \mathrm{~Hz}$. Moreover, this plate mode is also spatially coincident with cavity modes $q_{z}>0$ of frequency greater than $4700 \mathrm{~Hz}$. These cavity modes are highly non-resonant, but due to their spatial coincidences with the resonant plate modes, they play a significant role in the response of the present test case.

A second DMF calculation including non resonant modes of the cavity was performed. The modes of the cavity were chosen such that the modal orders $\left(q_{x}, q_{y}, q_{z}\right)$ verify:

$$
\left\{\begin{array}{l}
q_{x} \in\left[0, \bar{q}_{x}\right] \text { with } \bar{q}_{x}=\operatorname{int}\left[\max _{p \in[1, P]}\left[p_{x}\right]\right]+1, \\
q_{y} \in\left[0, \bar{q}_{y}\right] \text { with } \bar{q}_{y}=\operatorname{int}\left[\max _{p \in[1, P]}\left[p_{y}\right]\right]+1, \\
q_{z} \in\left[0, q_{z}^{\max }\right] .
\end{array}\right.
$$

Taking $q_{z}^{\max }=39,8400$ cavity modes were then considered and the highest frequency of these modes was $22213 \mathrm{~Hz}$. The results of this second DMF calculation are plotted by a dashed line in Fig. 4.

Contrary to the first DMF calculation including only the modes below $1200 \mathrm{~Hz}$, we observed good agreement between this second DMF calculation and the FE one for the receiving point on the plate as well as for the receiving point inside the cavity. The two curves (i.e. dashed and full) appear to be almost superimposed. This validates the convergence of the DMF for the present case. Moreover, these results point out that it is necessary to take into account the acoustic modes which coincide spatially with the structure modes, even if these acoustic modes are non resonant and are in a high frequency range. These modes are associated with the quasi-incompressibility of the fluid in [20] and are called the uncompressible acoustic modes. It is needed to take them into account in order to simulate the added mass effect of the heavy fluid. This point contrasts with the classical SEA assumption which only considers the resonant modes of the frequency band of excitation. In the next section, we will analyse the effect of these non resonant modes on the modal energy distribution of each subsystem. For the final validation of the DMF calculation for heavy fluid cases, a comparison with results published in the literature [20] is proposed in appendix A. 


\section{ANALYSIS OF THE MODAL INTERACTION FOR A BROAD BAND EXCITATION}

\subsection{Modal energy distribution}

Here we consider the test case defined in section 3.1. The excitation is a mechanical point force at $(0.3 \mathrm{~m}, 0.5 \mathrm{~m}, 1.4 \mathrm{~m})$ having a white noise spectrum in a given one third octave band $\left(S_{F F}=1 / 2 \pi \mathrm{N}^{2} /(\mathrm{rad} / \mathrm{s})\right)$. For the sake of clarity, numerical results are presented for a single one third octave band. Global energy results as a function of the third octave band will be presented in section 5 .

The one-third octave band considered is centred on $2500 \mathrm{~Hz}$. In this band, the plate has 86 resonant modes and the cavity has 175 resonant modes. Here, the modes are said to be "resonant" if their frequencies are contained in the one-third octave band. DMF calculations are performed by considering non-resonant modes as in section 3 . The plate and cavity modes contained in the enlarged frequency band $\left[0,1.5 f_{\max }\right]$ are taken into account where $f_{\max }$ is the upper bound of the one-third octave band (i.e. $f_{\max }=2825 \mathrm{~Hz}$ ). Moreover, the cavity modes conforming to the spatial coincidence conditions (26) are also taken into account. Overall, 500 plate modes and 30159 cavity modes are considered. Modal energies are calculated using Eqs. (20) and (21).

Modal Energy Distributions (MEDs) for the plate and the cavity are plotted as a function of the modal frequency in Fig. 6. The cut frequencies of the third octave are symbolised by vertical dashed lines.

It can be seen that the highest energy modes on the MED of the plate do not necessarily fall inside the frequency band of excitation. There is a frequency shift which could be attributed to an added mass effect of the fluid, as will be seen in section 4.2. Moreover, it can be observed that the first plate modes have a slightly higher energy level than modes closer to the frequency band of excitation. It will be seen later on that this is due to spatial coincidences of the first plate modes with the resonant cavity modes.

For the MED of the cavity, the highest energy modes are contained in the frequency band of excitation. However, we also observe that the energy of certain high frequency modes may be significant. If we consider equation (25) with $\tilde{f}_{p}=2500 \mathrm{~Hz}$, we obtain $\tilde{f}_{q}(0)=8442 \mathrm{~Hz}$ which is approximately the frequency above which the high frequency modes have significant energy. This seems to indicate that these modes are spatially coincident with the resonant plate modes. 


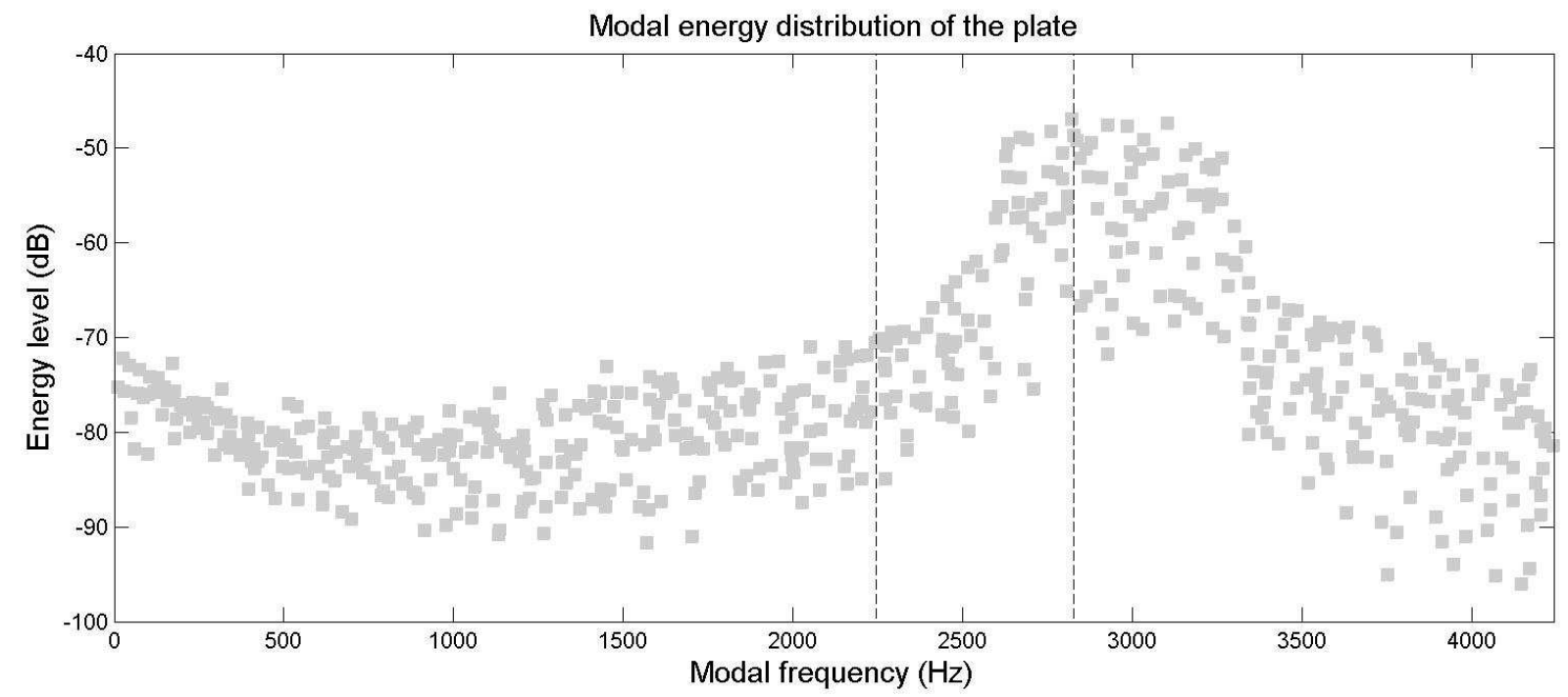

(a)

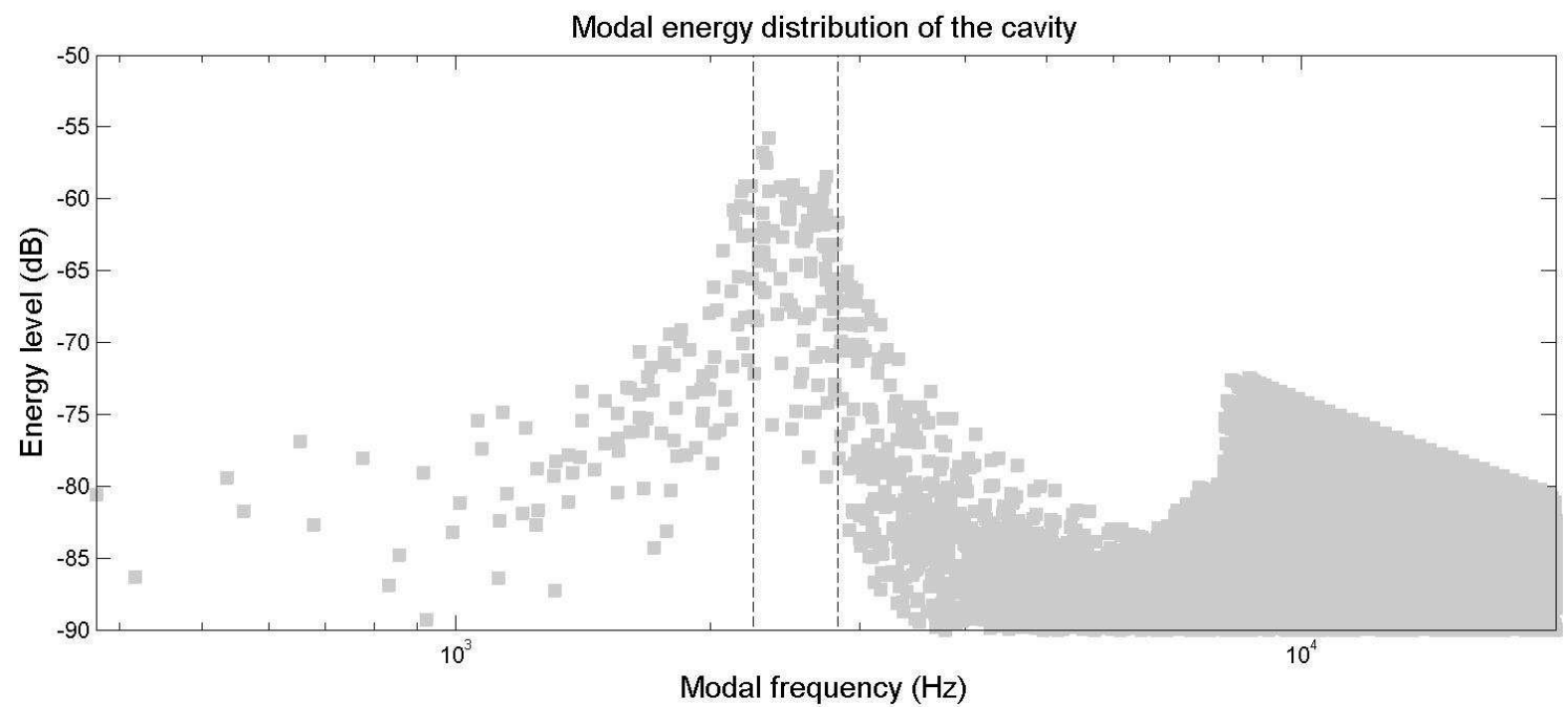

(b)

Fig. 6. Modal Energy Distribution: (a), for the plate; (b), for the cavity. White noise excitation in the third octave band of central frequency $2500 \mathrm{~Hz}$ (cut-off frequencies symbolised by vertical dashed line).

At this stage, we can underline that the total energy of the plate (resp. cavity) is not necessarily twice the kinetic energy (resp. strain energy) as assumed in SEA. For example, for the plate, is can be seen that for a first class of frequency modes well below the excitation frequencies, their strain energies are negligible compared to their kinetic energies while for a second class composed of frequency modes inside or close to the frequency band of excitation, their strain and kinetic energies have almost the same magnitudes. This difference can be easily explained by Eq. (20) and the difference between the modal frequency and the excitation frequencies. The result is that for the first class, the total energy (of a given mode) can be approximated by its kinetic energy while for the second class, the total energy can be approximated by twice its kinetic energy. This latter approximation is classically used in SEA. As pointed out here, it is valid only if the resonant modes contribute mainly to the subsystem responses. As observed in Fig. 6, the non resonant modes of the cavity can make a significant 
contribution, in which case conformity with the classical SEA approximation may not achieved for a heavy fluid cavity. For the present case, we obtain a kinetic energy of $-44.2 \mathrm{~dB}$ (ref. $1 \mathrm{~J}$ ) while the strain energy is $-39.7 \mathrm{~dB}$. An error of almost $2 \mathrm{~dB}$ can be introduced if we consider that the total energy is twice the strain energy $(-36.7 \mathrm{~dB}$ against $-38.7 \mathrm{~dB}$ for the true value). This emphasises the fact that particular attention should be paid to the relations between the different energies when the contribution of non resonant modes is significant.

\subsection{Added mass and added stiffness}

Certain observations were made in the previous section. We now investigate the DMF equations in order to analyze the modal interaction and find explanations for the previous observations.

\subsubsection{Theoretical developments}

Since a significant effect of the non resonant cavity modes is observed, we organise these modes into three groups $Q_{n r}^{-}, Q_{r}, Q_{n r}^{+}$depending on their angular frequencies:

$$
\left\{\begin{array}{l}
q \in Q_{n r}^{-} \Leftrightarrow \omega_{q} \in\left[0, \omega_{\min }(1-\alpha)[\right. \\
q \in Q_{r} \Leftrightarrow \omega_{q} \in\left[\omega_{\min }(1-\alpha), \omega_{\max }(1+\alpha)\right] \\
\left.q \in Q_{n r}^{+} \Leftrightarrow \omega_{q} \in\right] \omega_{\max }(1+\alpha),+\infty[
\end{array}\right.
$$

where $\omega_{\min }$ and $\omega_{\max }$ are, respectively, the lower and upper cut-off angular frequencies of the frequency band considered and $\alpha$ is a margin coefficient. In what follows, we set $\alpha=0.05$.

$Q_{r}$ is the set of resonant modes while $Q_{n r}^{-}, Q_{n r}^{+}$are the lower and upper sets of non resonant modes, respectively.

We introduce approximations in (14). To do this, we break down the matrices $Z_{22}$ and $W_{12}$ accordingly with the different sets of modes:

$$
Z_{22}=\left[\begin{array}{ccc}
Z_{22}^{n r-} & 0 & 0 \\
0 & Z_{22}^{r} & 0 \\
0 & 0 & Z_{22}^{n r+}
\end{array}\right], W_{12}=\left[\begin{array}{lll}
W_{12}^{n r-} & W_{12}^{r} & W_{12}^{n r+}
\end{array}\right] .
$$

With this decomposition, Eq. (14) can be written in the form

$$
\mathrm{X}_{1}=\left[Z_{11}-\omega^{2}\left(W_{12}^{n r-} Z_{22}^{n r--^{-1}} W_{12}^{n r-{ }^{T}}+W_{12}^{r} Z_{22}^{r-1} W_{12}^{r^{T}}+W_{12}^{n r+} Z_{22}^{n r-^{-1}} W_{12}^{n r+{ }^{T}}\right)\right]^{-1} F_{1} .
$$

Then, we introduce approximations of the modal impedance matrices $Z_{22}^{n r-}$ and $Z_{22}^{n r+}$ (with regard to the modal frequencies and the excitation frequencies)

$$
Z_{22}^{n r-} \approx \operatorname{diag}\left[-K_{q} \omega^{2}\right]_{q \in Q_{n r}^{-}}, Z_{22}^{n r+} \approx \operatorname{diag}\left[K_{q} \omega_{q}^{2}\right]_{q \in Q_{n r}^{+}},
$$

while $Z_{22}^{r}$ is unchanged (i.e. $Z_{22}^{r}=\operatorname{diag}\left[K_{q}\left(-\omega^{2}+j \omega \omega_{q} \eta_{2}+\omega_{q}^{2}\right)\right]_{q \in Q_{r}}$ ).

These approximations are based on the fact that the behaviour of a non-resonant mode of $Q_{n r}^{-}$ is mainly controlled by its mass while a non-resonant mode of $Q_{n r}^{+}$is controlled by its stiffness. 


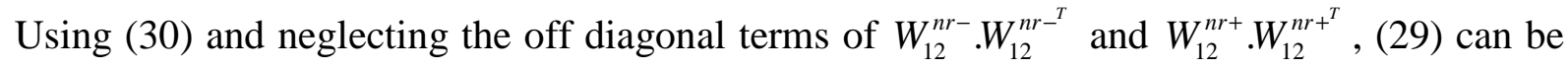
rewritten as

$$
\mathrm{X}_{1} \approx\left[\bar{Z}_{11}-\omega^{2} W_{12}^{r} Z_{22}^{r^{-1}} W_{12}^{r^{T}}\right]^{-1} F_{1}
$$

with $\bar{Z}_{11}=\operatorname{diag}\left[-\left(M_{p}+\bar{M}_{p}\right) \omega^{2}+j \omega M_{p} \omega_{p} \eta_{1}+\left(K_{p}+\bar{K}_{p}\right)\right]_{P \times P}$, and,

$$
\bar{M}_{p}=\sum_{q \in Q_{n r}^{+}} \frac{1}{K_{q}}\left(\frac{W_{p q}}{\omega_{q}}\right)^{2}, \bar{K}_{p}=\sum_{q \in Q_{n r}^{-}} \frac{\left(W_{p q}\right)^{2}}{K_{q}} .
$$

$\bar{M}_{p}$ and $\bar{K}_{p}$ represent an added mass and an added stiffness on the $p^{\text {th }}$ plate mode. The added mass is induced by the stiffness response of the non resonant modes $Q_{n r}^{+}$, and conversely, the added stiffness is due to the mass response of the non-resonant modes $Q_{n r}^{-}$.

We underline that added mass and added stiffness effects have already been highlighted in Ref. [20] for a plate coupled with a water filled cavity. These effects are related to incompressible acoustic modes for heavy fluids. In this reference, a $(\mathrm{u}, \phi)$ formulation (where $\phi$ is a fluid displacement potential) is considered and the static displacement potential is taken into account. This last term is evaluated by two techniques: a modal expansion on the acoustic modes and an analytical calculation available for a rectangular plate coupled to a parallelepipedic cavity. The first technique gives an approximate expression of the added mass matrix as a function of the acoustic mode shapes. The diagonal terms are similar to the added mass given by Eq. (33). A difference occurs on the modes retained in the summation. This can be explained by the fact that the added mass of the present paper represents only the effect of the non-resonant modes of frequency above the frequency band of excitation (i.e. modes of $Q_{n r}^{+}$) whereas the added mass defined in [20] represents the effect of "all" the acoustic modes (of the truncated set defined in [20]). It is more difficult to achieve a comparison of the added stiffness as the quantity defined in the present paper is directly related to the mode set $Q_{n r}^{-}$which depends strongly on the frequency band of excitation. Due to a difference of definition, this dependency of the added stiffness does not appear in [20].

From these added mass and added stiffness terms, a modified modal frequency can then be defined by

$$
\bar{\omega}_{p}=\sqrt{\frac{K_{p}+\bar{K}_{p}}{M_{p}+\bar{M}_{p}}}
$$

The response of the resonant cavity modes can be deduced with

$$
Y_{2} \approx-j \omega Z_{22}^{r^{-1}} W_{12}^{r^{T}} \mathrm{X}_{1} \text {. }
$$

Equations (31) and (35) can be interpreted as the results of the coupling of modified plate modes (taking the added mass and stiffness into account) with the resonant modes $Q_{r}$ of the cavity. In what follows, the model related to these two equations is called the "approximate DMF" whereas the model related to equations (14) and (15) is called the "basic DMF". With this approximate model, the energies of the non resonant modes of the cavity are not obtained directly because their amplitudes are not explicitly calculated. Their energies are, however, 
related to the kinetic energy associated with the added masses and the strain energy associated with the added stiffness. For example, the kinetic energy of the added masses is expressed by

$$
<\bar{E}_{1}^{K}>=\frac{1}{2} \sum_{p=1}^{P} \int_{\Delta \omega} \sum_{q \in Q_{n r}^{+}} \frac{1}{K_{q}}\left(\frac{W_{p q}}{\omega_{q}}\right)^{2} \omega^{2} S_{\chi_{p} \chi_{p}}(\omega) d \omega .
$$

On the other hand, the part of the cavity's kinetic energy represented by the cavity modes $Q_{n r}^{+}$ is obtained by summing the strain energy of these modes (in accordance with (18))

$$
<\bar{E}_{2}^{K}>=\sum_{q \in Q_{n r}^{+}}<E_{q}^{S}>=\frac{1}{2} \sum_{q \in Q_{n r}^{+}} \int_{\Delta \omega} K_{q} \omega_{q}^{2} S_{\zeta_{q} \zeta_{q}}(\omega) d \omega .
$$

The ASD of the amplitude of mode $q, S_{\zeta_{q} \zeta_{q}}$ can be related to the ASD of the modal amplitude of the plate $S_{\chi_{p} \chi_{p}}$ by considering the approximations (30) and neglecting the off diagonal terms of $W_{12}^{n r+} \cdot W_{12}^{n r+{ }^{T}}$ :

$$
S_{\zeta_{q} \zeta_{q}} \approx \frac{\omega^{2}}{\left(K_{q} \omega_{q}^{2}\right)^{2}} \sum_{p=1}^{P}\left(W_{p q}\right)^{2} S_{\chi_{p} \chi_{p}}, \forall q \in Q_{n r}^{+} .
$$

Introducing (38) in (37), we show that

$$
<\bar{E}_{2}^{K}>\approx<\bar{E}_{1}^{K}>\text {. }
$$

The part of the cavity's kinetic energy represented by the cavity modes $Q_{n r}^{+}$is therefore equal to the kinetic energy of the added masses. As (30) is assumed, the part of strain energy of the cavity represented by the same modes $Q_{n r}^{+}$is assumed negligible. Then, the part of the total energy of the cavity represented by the modes $Q_{n r}^{+}$is given by the kinetic energy of the added masses. Similar reasoning can be used for evaluating the part of the total energy of the cavity represented by the modes $Q_{n r}^{-}$from the strain energy associated with the added stiffness.

\subsubsection{Numerical application}

A numerical application of the previous developments is proposed in this section.

We consider the same case as in section 4.1. To illustrate the added mass effect, in Fig. 7a the normalised added mass $\bar{M}_{p} / M_{p}$ (see Eq. (33)) is plotted as a function of the plate modal order, $p$. These values can be compared to the normalised added mass calculated for an infinite thin plate loaded by a heavy fluid on one side, $M_{\infty}$ (see [17]):

$$
M_{\infty}(\omega)=\frac{\rho_{0}}{\rho h \sqrt{k_{f}(\omega)^{2}-k_{0}(\omega)^{2}}},
$$

where $k_{0}$ and $k_{f}$ are, respectively, the acoustic wavenumber of the heavy fluid and the flexural wavenumber of the plate at the angular frequency $\omega$. 
(a)

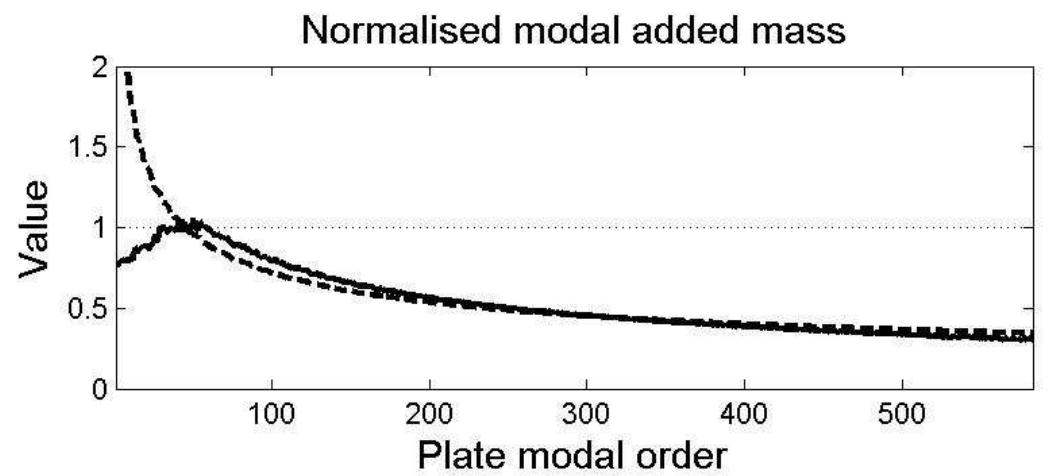

(b)

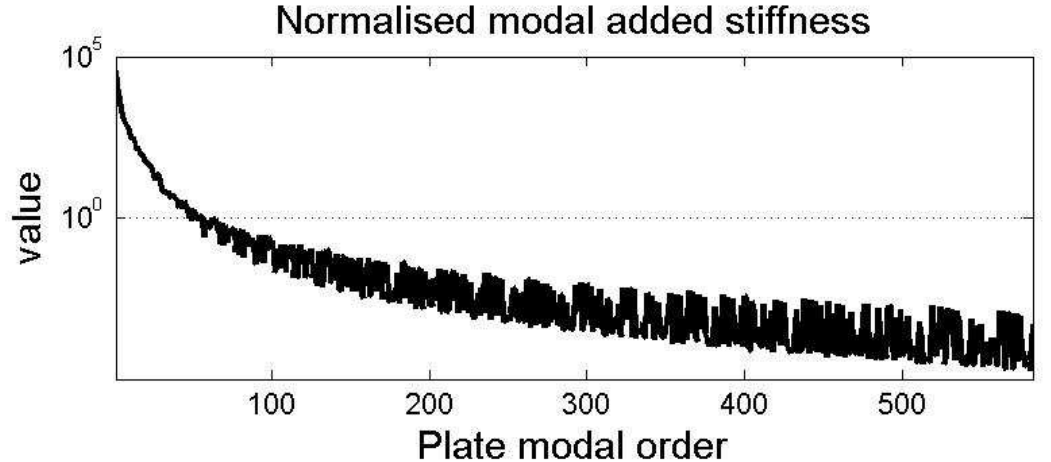

(c)

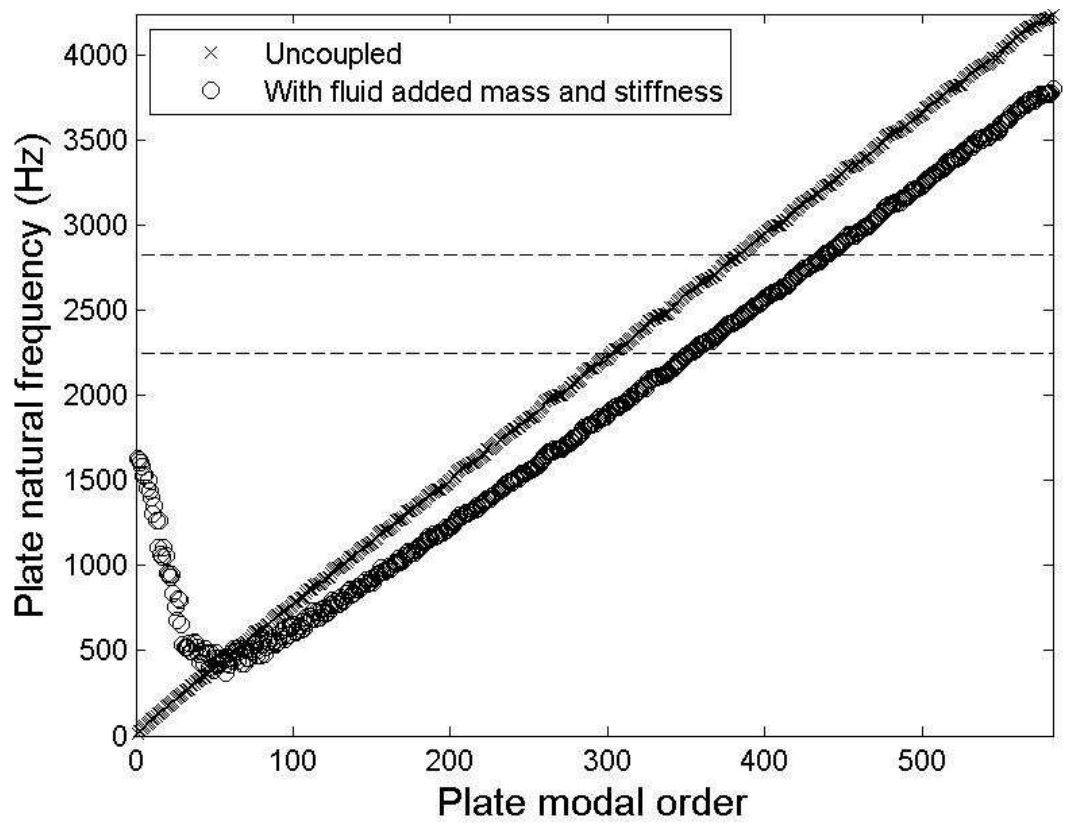

Fig. 7. Different results as a function of the plate modal order, $p$ : (a), full line, normalised modal added mass (i.e. $\bar{M}_{p} / M_{p}$ ); dashed line, added mass estimated from the infinite fluid loaded plate. (b), normalised modal added stiffness (i.e. $\bar{K}_{p} / K_{p}$ ); (c), plate natural frequency, with (circle) and without (cross) taking into account the mass and stiffness effects of the non-resonant cavity modes. Results for an excitation in the third octave band $2500 \mathrm{~Hz}$. 
The added mass $M_{\infty}\left(\omega_{p}\right)$ is plotted with a dashed line in Fig. 7a. We observe that for the modes above $400 \mathrm{~Hz}$, their normalised added masses can be approximated by those of the infinite plate at frequencies corresponding to the modal frequencies. For modes below 400 $\mathrm{Hz}$, the infinite plate model overestimates the added mass. This is certainly due to the fact that, contrary to the infinite plate, the cavity has a finite dimension in the direction perpendicular to the plate. The volume of the fluid moved by the wave motions of the plate (of large wavelengths) is limited by the sizes of the cavity. In other word, the added mass must normally be limited to the total mass of the fluid contained in the cavity, which is not infinite.

For the normalised added stiffness $\bar{K}_{p} / K_{p}$ plotted in Fig. 7b, the values decrease when the modal frequencies increase and they are significant (i.e. greater than 0.1) for frequencies below $1000 \mathrm{~Hz}$. Only the low-frequency non-resonant plate modes are then concerned by this effect for the present case. As already mentioned above, we remember that this added stiffness is directly related to the frequency band of excitation. It represents the effect of the non resonant acoustic modes below the frequency band of excitation. If the latter changes, the added stiffness may change.

These two effects lead to a modification of the modal frequencies (34): the added stiffness tends to increase the frequency whereas the added mass tends to decrease it. We compare the initial modal angular frequency $\omega_{p}$ with the modified one $\bar{\omega}_{p}$ in Fig. 7c. The added stiffness significantly increases the frequencies of the "first" plate modes which remain outside the frequency band of excitation. These plate modes remain non-resonant. For these "first" modes, the added stiffness effect is greater than the added mass effect, which explains why $\bar{\omega}_{p}$ is greater than $\omega_{p}$.

The other plate modes are more influenced by the added mass than by the added stiffness. Their modal frequencies decrease compared to the initial ones. If we plot the plate's MED as a function of the modified modal angular frequency $\bar{\omega}_{p}$ (not shown here), we observe that the highest energy modes are contained in the frequency band of excitation (contrary to Fig. 6 for which the MED was plotted as a function of the initial modal frequency). The added mass due to the non resonant modes of cavity $Q_{n r}^{+}$explains the frequency shift observed previously in section 4.1 (i.e. Fig. 6a).

In order to validate the approximations used to obtain the approximate DMF model (i.e. Eqs. (31) and (35)), we compare in Fig. 8 the MEDs obtained with the basic and the approximate models. Globally, the MEDs are correctly described by the approximate model. However, some discrepancies can be observed. They vary from one mode to another and are caused by the fact that we have ignored the direct coupling between the plate modes introduced by the non resonant modes of the cavity (i.e. the diagonal terms of $W_{12}^{n r-} \cdot W_{12}^{n r^{T}}$ and $W_{12}^{n r+} \cdot W_{12}^{n r+{ }^{T}}$ neglected). 


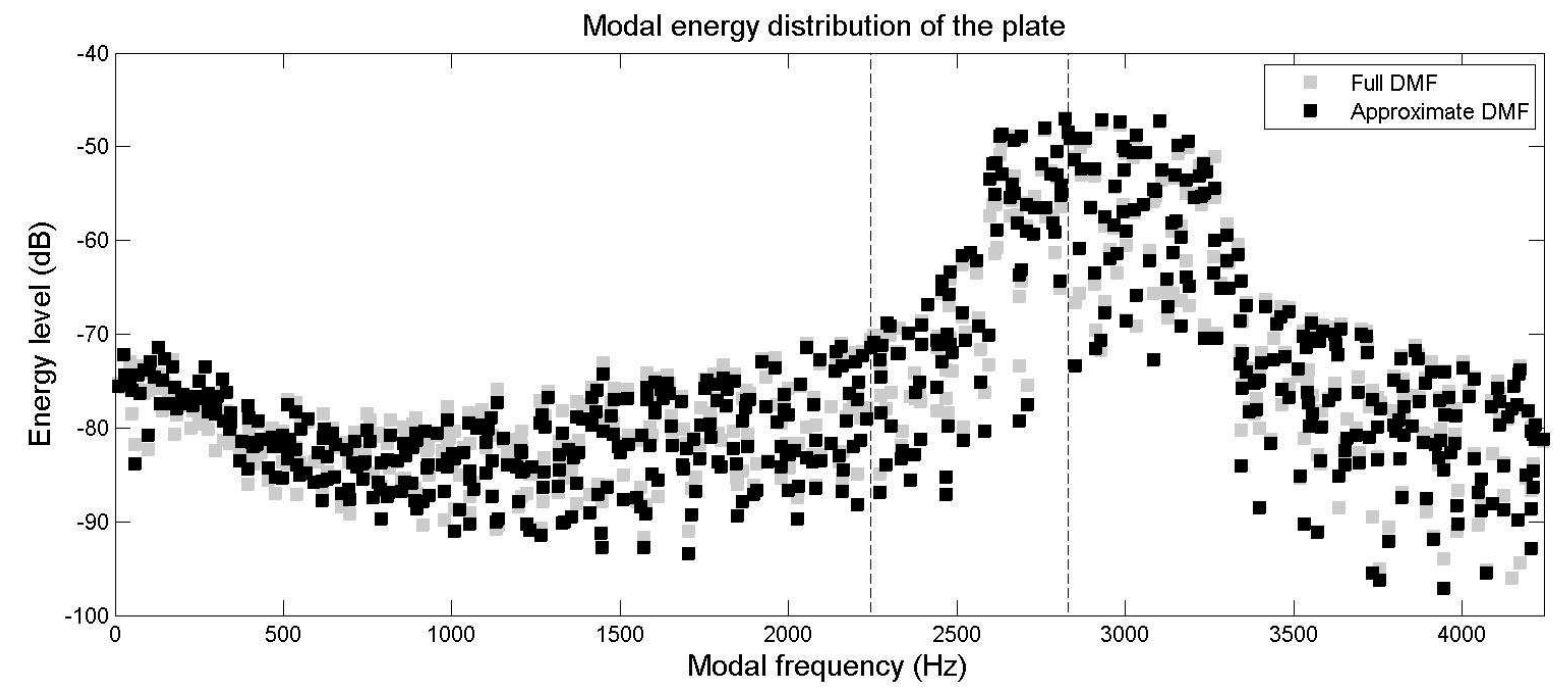

(a)

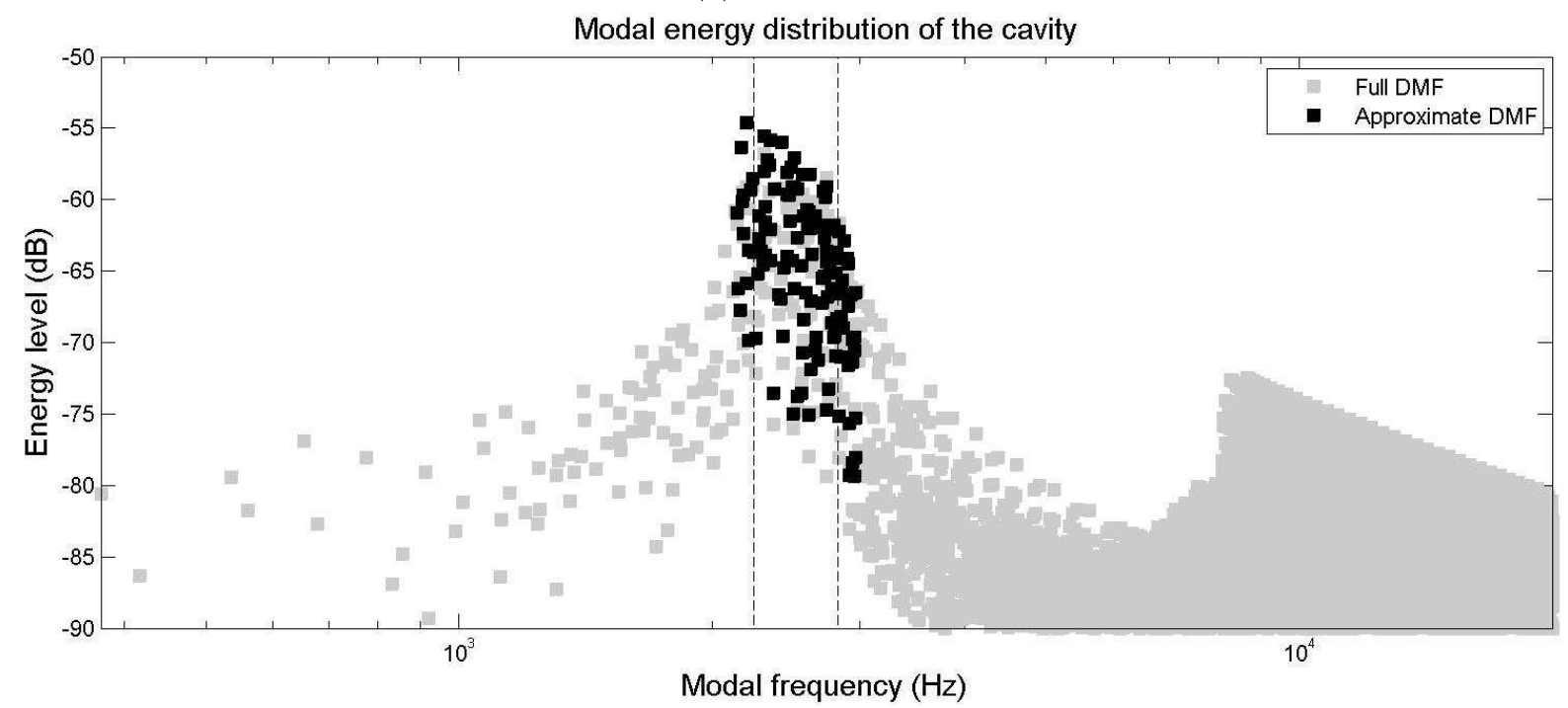

(b)

Fig. 8. Modal Energy Distribution: (a), for the plate; (b), for the cavity. Comparison of two results: grey squares, a basic DMF calculation; black squares, an approximate DMF calculation describing the non resonant mode of the cavity by their mass and stiffness effect.

White noise excitation in the $2500 \mathrm{~Hz}$ third octave band (cut-off frequencies of this band symbolised by vertical dashed line).

These discrepancies have little influence however on the estimation of the total energy of the plate and the cavity. Indeed, for the plate, we obtain an energy level of $-33.7 \mathrm{~dB}$ (ref. $1 \mathrm{~J}$ ) with the basic model versus $-33.5 \mathrm{~dB}$ with the approximate model. For the cavity, the comparison can be performed for:

- (a), the resonant energy of the cavity (which corresponds to the energy of the resonance cavity modes). The basic model gives $-41.8 \mathrm{~dB}$ against $-41.1 \mathrm{~dB}$ for the approximate one;

- (b), the total energy of the cavity. In this case, the result of the approximate model is obtained by adding the resonant energy of the cavity with the energies of the added masses and added stiffness (which represent the energies of the non resonant 
cavity modes as developed in section 4.2.1). The "basic" model gives $-38.7 \mathrm{~dB}$ against $-38.5 \mathrm{~dB}$ for the approximate one.

These energy results given by the "approximate" DMF model are fully satisfactory and validate the assumptions made for this case. Moreover, the comparison of the resonant energy with the total energy of the cavity $(-41.8 \mathrm{~dB}$ with $-38.7 \mathrm{~dB})$ shows here that the non resonant modes of the cavity significantly contribute to its energy response.

\subsection{Influence of the coupling of non resonant plate modes in spatial coincidence with the resonant cavity modes}

In Fig. 6a we saw that many non resonant plate modes have a low energy level compared to the highest energy modes. Thus these modes have a potentially negligible effect on the response of the plate-cavity system. In this section, we study the influence of these non resonant modes of the plate on the energy response of the plate and the cavity. We define the set of resonant modes $P_{r}$ and non-resonant modes $P_{n r}^{-}, P_{n r}^{+}$accordingly with the modified modal frequencies, $\bar{\omega}_{p}$ :

$$
\left\{\begin{array}{l}
p \in P_{n r}^{-} \Leftrightarrow \bar{\omega}_{p} \in\left[0, \omega_{\min }(1-\alpha)[\right. \\
p \in P_{r} \Leftrightarrow \bar{\omega}_{p} \in\left[\omega_{\min }(1-\alpha), \omega_{\max }(1+\alpha)\right] \\
\left.p \in P_{n r}^{+} \Leftrightarrow \bar{\omega}_{p} \in\right] \omega_{\max }(1+\alpha),+\infty[
\end{array}\right.
$$

DMF calculations without the non-resonant modes $P_{n r}^{+}$show that these modes have a negligible effect on the response of the plate and the cavity. This could be expected as these modes are excited little by the external force and they are not strongly coupled with the resonant cavity modes, since they are not in a space or frequency coincident with these modes.

Conversely, DMF calculations without the non-resonant plate modes $P_{n r}^{-}$show that these modes have a significant effect on the total energy of the cavity (while the energy of the plate is not influenced). For the same case as Fig. 6, we compared the MED of the resonant modes of the cavity for two DMF calculations: one with and the other without modes $P_{n r}^{-}$(results not plotted here). We observed that the second calculation almost systematically overestimates the energy of the cavity modes. It gives a total energy for the resonant modes of $-39.2 \mathrm{~dB}$ against $-41.8 \mathrm{~dB}$ for the first calculation. Some modes of the set $P_{n r}^{-}$therefore play the role of pumping the energy of the cavity modes. By studying the significant intermodal work in Fig. 5, the "first" plate modes (i.e. modes of frequency below $500 \mathrm{~Hz}$ ) appear to coincide in space with the resonant cavity modes. Although these "first" plate modes and the resonant cavity have very different frequencies, they can exchange significant energy due to their spatial coincidence. The response of these plate modes results from an equilibrium between the power flows from the external excitation and the resonant cavity modes. In the present case, this equilibrium leads to significant power exchanged from the resonant cavity modes to the "first" plate modes. Part of the energy of the resonant cavity modes is then pumped by the first non resonant plate modes. This also explains why we noticed in Fig. 6 that the "first" plate modes have more pronounced energy level than "higher" modes which are nevertheless closer to the band of excitation. 


\subsection{Synthesis of the modal interaction}

On the basis of the previous discussions, Fig. 9 represents the modal coupling scheme for an excited plate coupled with a heavy fluid cavity. We show that the behaviour of the cavity's non-resonant modes can be represented by added masses and stiffness on the plate modes. This results in a modification of the modal frequency of these plate modes. The resonant plate modes can then defined in relation to the modified modal frequency and the plate modes above the frequency band of excitation can be ignored. Conversely, the non-resonant plate modes in space coincident with the resonant cavity modes can participate significantly in the power exchanged between the structure and the cavity and they cannot be neglected in general.

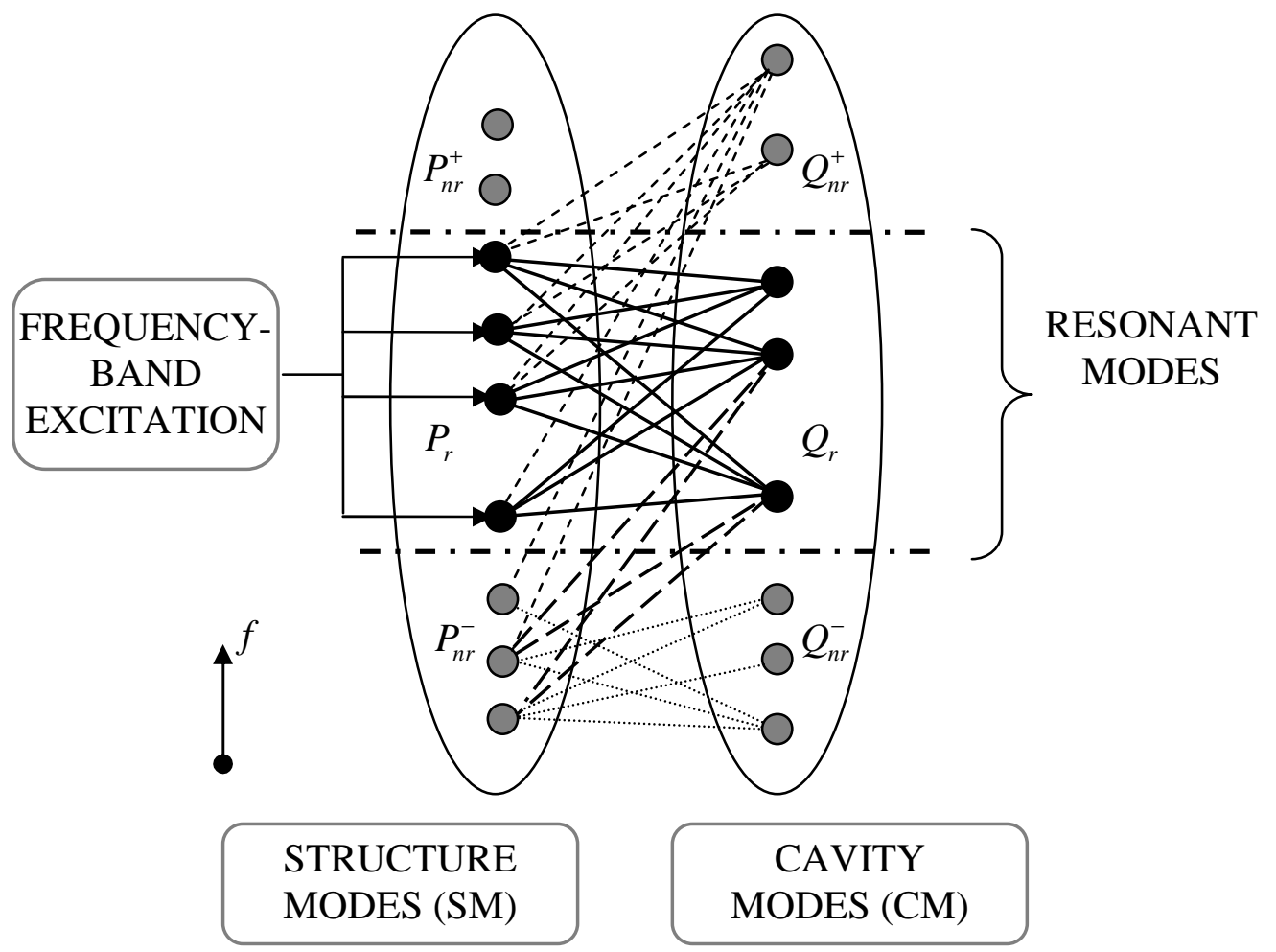

- Resonant coupling between CM and SM

Added mass effect of CM on SM

Added stiffness effect of CM on SM

- - - Spatial coupling of resonant CM with SM

Fig. 9. Illustration of the modal coupling scheme for an excited structure filled by a heavy fluid. 


\section{FREQUENCY RESPONSE OF THE PLATE/WATER-FILLED CAVITY SYSTEM}

In this section, we analyse the energy response given by different calculations for one third octave band excitations from $500 \mathrm{~Hz}$ to $16 \mathrm{kHz}$. Three DMF calculations are considered:

- A "basic" DMF calculation as defined in section 3. It takes into account the non resonant cavity modes. It constitutes the reference here;

- An "approximate" DMF calculation as defined previously in section 4.

- A "resonant" DMF calculation which takes only the resonant modes of the "approximate" DMF model into account.

For each third octave band, Tab. 1 gives the total mode number taken into account by the "basic" DMF calculation and the number of resonant modes of each subsystem.

\begin{tabular}{|c|c|c|c|c|c|c|c|c|}
\hline & $500 \mathrm{~Hz}$ & $630 \mathrm{~Hz}$ & $800 \mathrm{~Hz}$ & $1000 \mathrm{~Hz}$ & $1250 \mathrm{~Hz}$ & $1600 \mathrm{~Hz}$ & $2000 \mathrm{~Hz}$ & $2500 \mathrm{~Hz}$ \\
\hline$N_{1}^{\text {Total }}$ & 91 & 118 & 151 & 193 & 244 & 311 & 394 & 500 \\
\hline$N_{1}^{\text {Resonant }}$ & 16 & 22 & 29 & 35 & 44 & 56 & 68 & 86 \\
\hline$N_{2}^{\text {Total }}$ & 6719 & 8399 & 10199 & 12919 & 15959 & 19319 & 23919 & 30159 \\
\hline$N_{2}^{\text {Resonant }}$ & 2 & 5 & 7 & 13 & 26 & 47 & 91 & 175 \\
\hline & $3150 \mathrm{~Hz}$ & $4000 \mathrm{~Hz}$ & $5000 \mathrm{~Hz}$ & $6300 \mathrm{~Hz}$ & $8000 \mathrm{~Hz}$ & $10 \mathrm{kHz}$ & $12.5 \mathrm{kHz}$ & $16 \mathrm{kHz}$ \\
\hline$N_{1}^{\text {Total }}$ & 635 & 803 & 1015 & 1186 & 1628 & 2056 & 2599 & 3277 \\
\hline$N_{1}^{\text {Resonant }}$ & 111 & 134 & 167 & 217 & 264 & 340 & 420 & 537 \\
\hline$N_{2}^{\text {Total }}$ & 37119 & 46079 & 57599 & 71999 & 89999 & 114239 & 143639 & 177484 \\
\hline$N_{2}^{\text {Resonant }}$ & 332 & 629 & 1235 & 2406 & 4737 & 9324 & 18409 & 36444 \\
\hline
\end{tabular}

Tab. 1. Mode number for each third octave band: $N_{1}^{\text {Total }}$ and $N_{2}^{\text {Total }}$, mode numbers for the basic DMF calculation for the plate and the cavity, respectively; $N_{1}^{\text {Resonant }}$ and $N_{2}^{\text {Resonant }}$, resonant mode numbers for the plate and the cavity, respectively.

A SEA calculation is also performed to compare its results to the DMF ones. Details of the SEA model are given in appendix C. An added mass effect of the fluid is taken into account in the calculation of the coupling loss factor and the plate modal density through an effective mass density ([17]). We underline that the SEA method gives us the total energy of each subsystem represented by their resonant modes. The energies related to non-resonant modes are not evaluated by this model. The SEA results are given here to evaluate its accuracy. They do not constitute a reference as we know that some of its assumptions (i.e. weak coupling, resonant transmission) are not fully respected. Comparison with the "basic" DMF which can be considered as the reference allows us to evaluate the errors induced by the non respect of the SEA assumptions.

Tab. 1 indicates that the number of resonant modes of the two subsystems is higher than 6 from the third octave band, $800 \mathrm{~Hz}$. This is a condition of validity of the SEA method. However, the SEA results are plotted for frequencies below this limit frequency as an indication. 
The total energy of the plate was evaluated for each third octave band by the four calculations described above. They give very similar results (not plotted here): an energy level of around $34 \mathrm{~dB}$ for each third octave. The "resonant" DMF and SEA calculations work. This indicates that the resonant modes of the plate are sufficient for describing the energy response of the plate. This could be expected as the plate is directly excited by the external force. On the contrary, in Fig. 10a, the results for the resonant energy of the cavity depend on the calculations. The "approximate" DMF model gives the same tendency as the "basic" DMF. Some discrepancies can be observed when the cavity has few resonant modes. This can be explained by the fact that cross terms were neglected in Eq. (31). On the other hand, it can be observed that the "resonant" DMF model deviates significantly from the "basic" DMF and "approximate" DMF ones. The difference between the "resonant" DMF and the "approximate" DMF lies in the non resonant plate modes which are not taken into account in the first model. Therefore, the discrepancies observed in Fig. 10a may be attributed to the contribution of the non resonant plate modes in spatial coincidence with the resonant modes of the cavity. For frequencies lower than $8 \mathrm{kHz}$, these plate modes pump part of the energies of the resonant cavity modes, whereas for higher frequencies, they contribute part of their energy to the cavity modes. This behaviour cannot be described by classical SEA which gives results similar to those of the "resonant" DMF model. Indeed, as SEA is based on a fundamental relation established for two oscillators excited by white noise forces [6], it can only describe the energy sharing between resonant modes. For the phenomenon of interest here, the energy is shared between the resonant acoustic modes and the non resonant structure modes. The fundamental SEA relation cannot be used to describe these exchanges.

The total energy of the cavity (taking the non resonant contributions into account) is proposed in Fig. 11a. The "basic" DMF and "approximate" DMF give very close results. The discrepancies observed in Fig. 10a in the lower part of the frequency domain are not found here because the non resonant contributions are dominant at these frequencies and are correctly described by the "approximate" DMF model (from the energies of the added masses and stiffness). By comparing the results of Figs. 10a and 11a, it can be seen that the non resonant modes have a significant contribution up to about $5 \mathrm{kHz}$.

To complete this discussion, we perform a second set of calculations considering a damped plate with an internal loss factor of $10 \%$ (i.e. $\eta_{1}=0.10$ ). The different models give similar results for the plate (not plotted here). The plate energy decreases between $8.5 \mathrm{~dB}$ and $10 \mathrm{~dB}$ compared to the previous case. At first sight, we can expect a decrease of exactly $10 \mathrm{~dB}$. Indeed, if we consider the SEA energy balance (C.1), plate energy $E_{1}$ can be approximated to $P_{i n j} / \omega_{c} \eta_{1}$ by neglecting the power flow between the plate and the cavity compared to the input power. Then, if the plate damping loss factor is multiplied by 10 , the plate energy should theoretically be divided by 10 . The approximation made previously is however not fully satisfied for this case with a heavy fluid cavity, explaining why a value of exactly $10 \mathrm{~dB}$ is not obtained. 
(a)

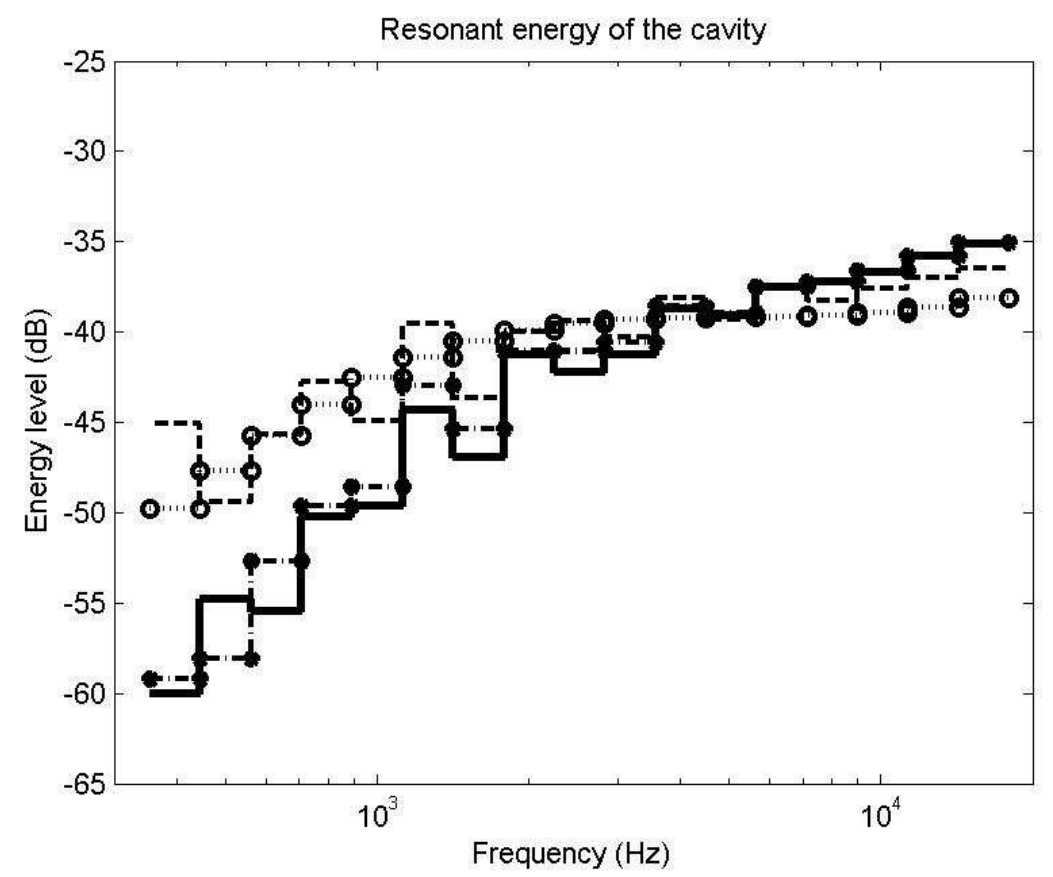

(b)

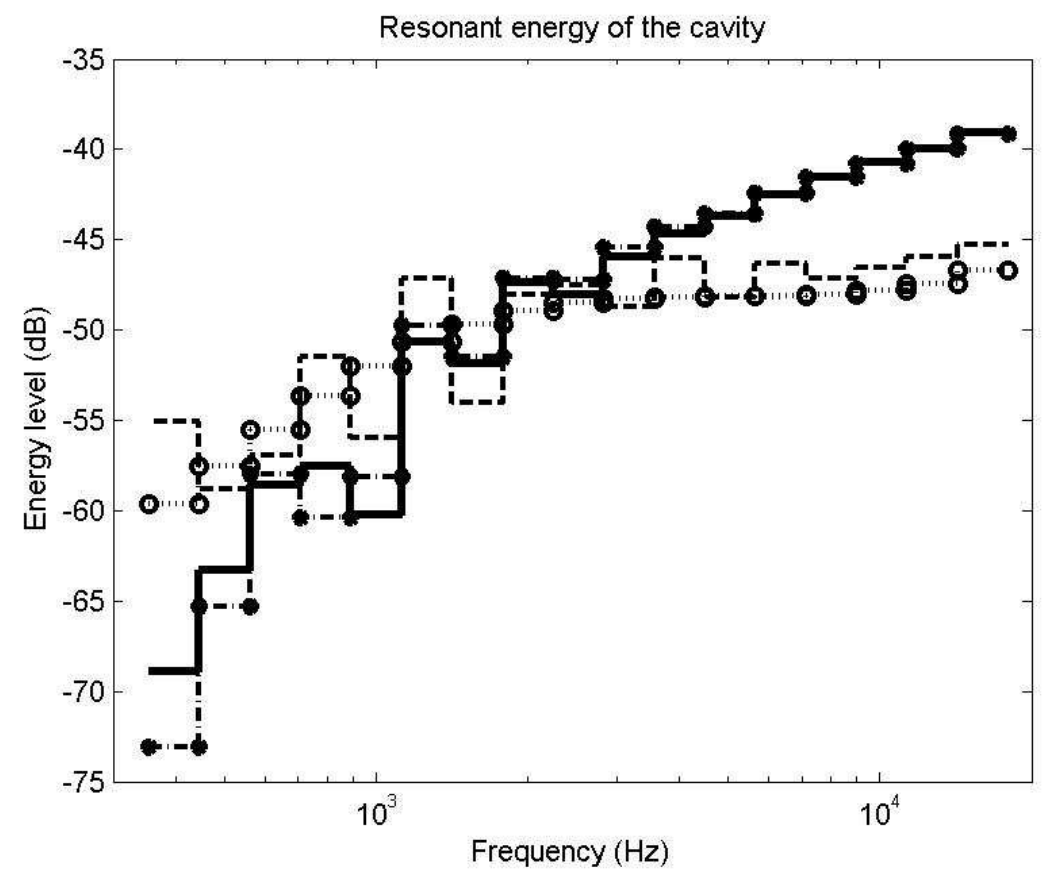

Fig. 10. Resonant energy of the cavity. Third octave band results.

Damping loss factor of the plate, $\eta_{1}=0.01$. Four calculations: Solid, basic DMF (reference);

Dashed-dotted (with cross), approximate DMF; dashed, resonant DMF; dotted (with circle), SEA. 
(a)

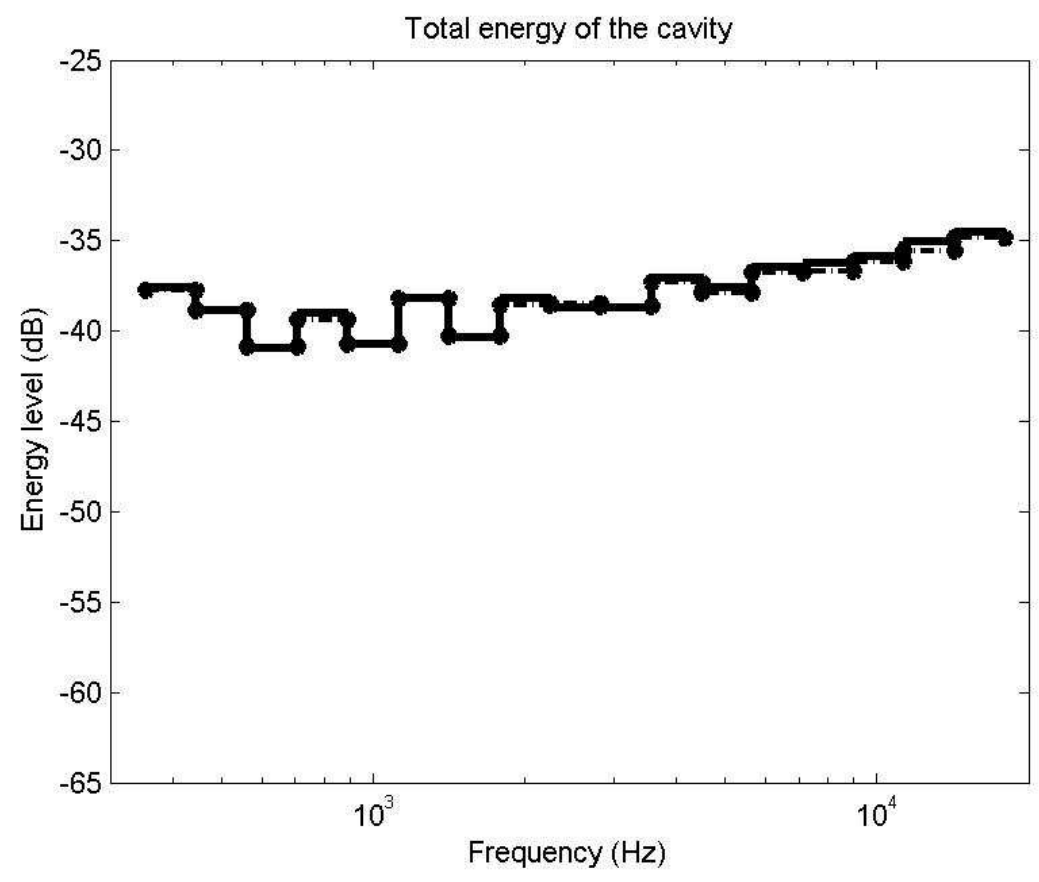

(b)

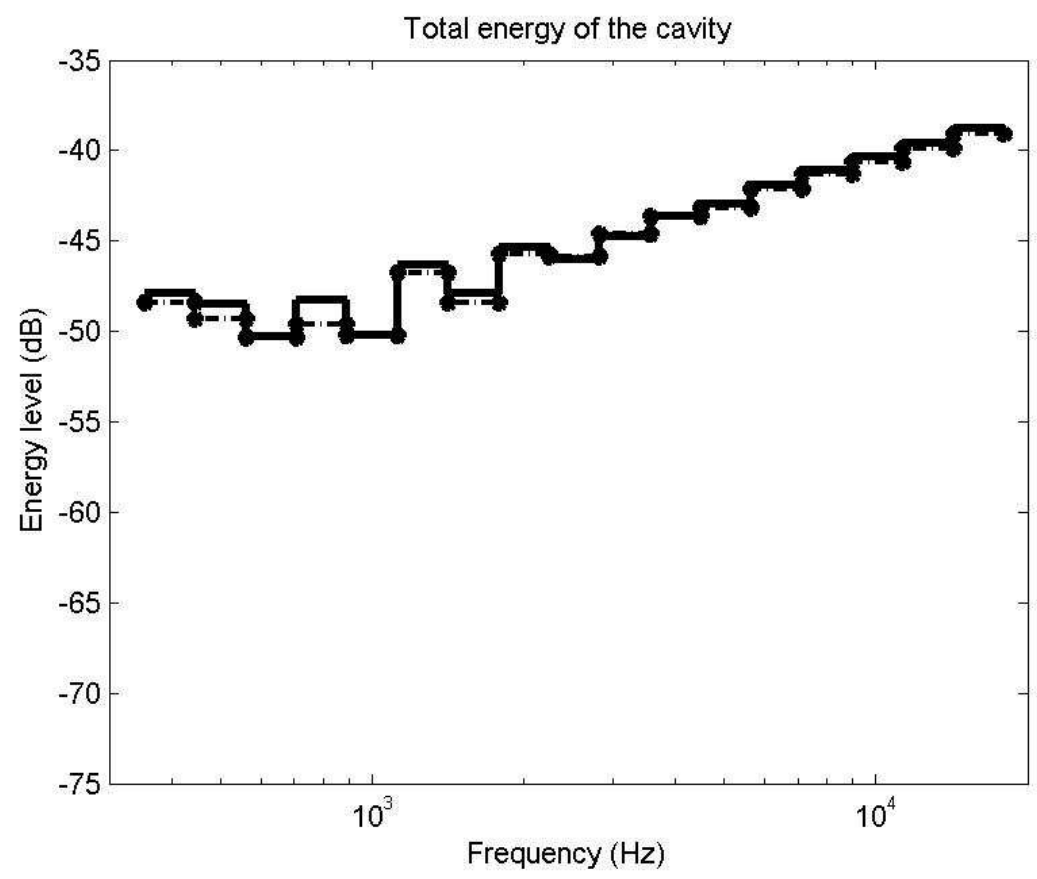

Fig. 11. Total energy of the cavity. Third octave band results.

Damping loss factor of the plate: (a), $1 \%$ (i.e. $\eta_{1}=0.01$ ); (b), $10 \%$ (i.e. $\eta_{1}=0.1$ ). Four calculations: Solid, basic DMF (reference); dashed-dotted (with cross), approximate DMF; dashed, resonant DMF;

Fig. 10b shows the resonant energy of the cavity. The "approximate" DMF always gives satisfactory results. The discrepancies between this model and the "resonant" DMF are larger here than for the case of Fig. 10a. The SEA calculation also gives the same tendency as the "resonant" DMF. Again, these two calculations cannot describe the energy exchanges between the non resonant plate modes and the resonant cavity modes which are in spatial 
coincidence. These exchanges of energy appear greater when the plate damping increases. It can be noticed that the non resonant plate modes pump part of the resonant energy of the cavity for frequencies lower than $1.6 \mathrm{kHz}$ and they contribute part of their energies for frequencies above this transitional frequency. The value of the transitional frequency has changed compared to the case of Fig. 10a (i.e. $1.6 \mathrm{kHz}$ against $8 \mathrm{kHz}$ ). The equilibrium of the non resonant plate modes resulting from the interaction with the resonant cavity modes and the external force has changed. Indeed, increased plate damping directly induces a decrease of the energy of the resonant plate modes, and then of the resonant cavity modes (in frequency coincidence), whereas the energy of the non resonant plate modes is not significantly modified. The energy ratio between the non resonant plate modes and the resonant cavity modes is then modified when the damping increases, which may explain a change of the energy equilibrium.

Fig. $11 \mathrm{~b}$ gives the total energy of the cavity and shows good agreement between the "basic" DMF and "approximate" DMF calculations. Comparison of the results of Fig. 10b and 11b indicate that multiplying the plate damping by 10 does not lead to the same decrease of the cavity energy as the plate energy (i.e. between $8.5 \mathrm{~dB}$ and $10 \mathrm{~dB}$ ). In the "high" frequencies, the decrease of cavity energy is limited to $4 \mathrm{~dB}$. This behaviour results from the significant contribution of the non resonant plate modes which are not influenced by the damping. The SEA method cannot represent this effect because it describes only the energy sharing between the resonant modes. If we consider the SEA energy balance again (i.e. Eq. (C.1)), the energy ratio $E_{2} / E_{1}$ is given by $\eta_{12} / \eta_{2}+\eta_{21}$ which is independent from the plate damping loss factor. The SEA method therefore gives the same decrease of energy for the plate and the cavity when the plate damping loss factor increases. This is in contradiction with the "basic" DMF results which can be considered as the reference. Thus, the SEA method fails for the present case.

In conclusion, the results presented in this section have shown that:

(a) the "approximate" DMF gives results in accordance with the "basic" DMF;

(b) SEA and "resonant" DMF models do not permit accurately describing the energy response of the cavity. The energy exchanges between the non resonant plate modes and the resonant cavity modes in spatial coincidence play a significant role that is not described by these two models;

(c) The increase of the plate damping for vibration and noise reduction is less efficient for the cavity energy than for the plate energy. The non resonant plate modes which are not influenced by the damping play an important role on this phenomenon.

\section{CONCLUSIONS}

The modal equations resulting from the dual modal formulation were considered in this paper in order to study the interaction of an excited vibrating structure coupled with a heavy fluid cavity. This formulation is used classically for a light fluid and the SEA formulation is also valid for a heavy fluid if enough modes are taken into account. A numerical study on a test case showed that convergence of the modal decompositions is possible if the non resonant cavity modes in spatial coincidence with the plate modes are sufficiently taken into account. The number of cavity modes necessary in the model can become significant. Analysis of the modal equations showed that the non-resonant cavity modes induce added masses and added stiffness on the plate modes which can be evaluated from the intermodal work. 
The modal added masses were compared to the added mass induced by a semi-infinite fluid domain loading an infinite plate. Due to the finite size of the cavity in the direction perpendicular to the plate, we observed differences for the "first" modes (having a large modal wavelength). The semi-infinite added mass model is not valid for angular frequency $\omega=0$ (it tends towards infinity). Normally, the added mass must be limited by the total mass of the fluid contained in the cavity which is not infinite. For higher modes, the infinite plate model gave a correct estimation of the modal added masses. Accurate estimations of the added mass and added stiffness are proposed in [20] from the knowledge of the static displacement potential. The interest of using the explicit expressions given in [20] could be investigated in the future for the present approach.

An "approximate" DMF model describing the non resonant modes of the cavity by their added masses and stiffness was proposed. This model was validated by different comparisons with the "basic" DMF model.

In some situations, we observed that the part of the total energy of the cavity represented by its resonant modes can be lower than the part due to its non-resonant modes. This second part of the cavity energy can be estimated from the kinetic energy of the added masses and the strain energy of the added stiffness on the plate modes. The "approximate" DMF model can then estimate this part of the energy even if it does not explicitly estimate the response of the non resonant cavity modes.

Moreover, the results highlighted a significant role played by the non resonant plate modes in spatial coincidence with the resonant cavity modes. Depending on the energy equilibrium, these modes can give energy to, or take it from, the resonant cavity modes. Due to this phenomenon and the weak influence of damping on the non resonant modes, increasing plate damping is less efficient on the cavity energy than on the plate energy. As classical SEA does not take these non-resonant modes into account, it cannot describe this behaviour correctly. Attention should be given to this point in the future in order to develop a SEA model dedicated to the heavy fluid case. The "approximate" DMF model is a good starting point for these developments. For a system with a complex geometry, the evaluation of the added masses and stiffness of the "approximate" DMF do not seem to be a problem. Indeed, the intermodal work can be estimated using finite element models for the low frequency modes $[13,23]$ while the infinite fluid loaded plate model can be used as an approximation of the added mass for the higher frequency modes. The main difficulty will consist in establishing the relation of energy exchanged between the non resonant structure modes with the resonant cavity modes. Indeed, the classical SEA relation established for two coupled oscillators assumes that the oscillators are excited by white noise excitation. Their responses are dominated by their resonances which is not the case for the non resonant structure modes. A study to elucidate this point should be performed in the future.

\section{ACKNOWLEDGMENTS}

This work was performed within the framework of the Labex CeLyA of Université de Lyon, operated by the French National Research Agency (ANR-10-LABX-0060/ ANR-11-IDEX0007). The author would like to thanks M. Jean-Michel David who has kindly sent him the numerical results of Refs. [19,20].

\section{REFERENCES}

[1] K.S. Sum, J. Pan, A study of the medium frequency response of sound field in a panel-cavity system, Journal of the Acoustical Society of America, 103 (1998) 1510-1519. 
[2] K.S. Sum, J. Pan, On acoustic and structural modal cross-couplings in plate-cavity systems, Journal of the Acoustical Society of America, 107 (2000) 2021-2038.

[3] J. Pan, D.A. Bies, The effect of fluid-structural coupling on acoustical decays in a reverberation room in the high-frequency range, Journal of the Acoustical Society of America, 87 (1990) 718-727.

[4] R. Ohayon, C. Soize, Structural Acoustics and Vibration, Academic Press, New York, 1998.

[5] K. Renji, P.S. Nair, S. Narayanan, Non-resonant response using statistical energy analysis, Journal of Sound and Vibration, 241 (2001) 253-270.

[6] R.H. Lyon, R.G. Dejong, Theory and application of statistical energy analysis, Second ed. - Boston: Butterworth-Heinemann, 1995. 277p.

[7] F. Fahy, Vibration of containing structures by sound in the contained fluid. Journal of Sound and Vibration, 10 (1969) 490-512.

[8] F. Fahy, Response of a cylinder to random sound in the contained fluid, Journal of Sound and Vibration, 13 (1970) 171-194.

[9] Y. Lei, J. Pan, M.P. Shang, Investigation of structural response and noise reduction of an acoustical enclosure using SEA method. Applied Acoustics, 73 (2012) 348355.

[10] F. Fahy, Sound and structural vibration, radiation, transmission and response, Academic press, London, 1985

[11] S. Finnveden, A quantitative criterion validating coupling power proportionality in statistical energy analysis, Journal of Sound and Vibration, 330 (2011) 87-109.

[12] A. Le Bot, V. Cotoni, Validity diagrams of statistical energy analysis, Journal of Sound and Vibration, 329 (2010) 537-554.

[13] N. Totaro, C. Dodard, J.L. Guyader, SEA coupling loss factors of complex vibroacoustic systems, Transaction of the ASME: Journal of Vibration and Acoustics, 131, 041009-1 (2009).

[14] A. Culla, A. Sestieri, Is it possible to treat confidentially SEA the wolf in sheep's clothing?, Mechanical systems and signal processing, 20 (2006) 1372-1399.

[15] L. Maxit, J.L. Guyader, Extension of SEA model to subsystem with non-uniform modal energy distribution, Journal of Sound and Vibration, 265 (2003) 337-358.

[16] G. Maidanik, Domed Sonar system, Journal of the Acoustical Society of America, 44 (1967) 113-124.

[17] W. Zhang, A. Wang, N. Vlahopoulos, K. Wu, High-frequency vibration analysis of thin elastic plates under heavy fluid loading by an energy finite element formulation, Journal of Sound and Vibration, 263 (2003) 21-46.

[18] M. Liu, A.J. Keane, R.E. Taylor, Modelling liquid-structure interactions within the framework of statistical energy analysis, Journal of Sound and Vibration, 238 (2000) 547-574.

[19] J.M. David, M. Menelle, Validation of a medium-frequency computational method for the coupling between a plate and a water-filled cavity, Journal of Sound and Vibration, 265 (2003) 841-861.

[20] J.M. David, M. Menelle, Validation of a modal method by use of an appropriate static potential for a plate coupled to a water-filled cavity, Journal of Sound and Vibration, 301 (2007) 739-759.

[21] E.H. Dowell, G.F. Gorman III, D.A. Smith, Acoustoelasticity: general theory, acoustic natural modes and forced response to sinusoidal excitation, including comparisons with experiment, Journal of Sound and Vibration, 52 (1977) 519-542.

[22] B. Tabarrok, Dual formulations for acousto-structural vibrations, International Journal for Numerical Methods in Engineering, 13 (1978) 197-201 
[23] L. Maxit, J.-L. Guyader, Estimation of SEA coupling loss factors using a dual formulation and FEM modal information. Part I: Theory, Journal of Sound and Vibration, 5, (2001) 907-930.

[24] S.H. Crandal, W.D. Mark, Random vibration in mechanical system, Academic press, New York, 1963, 166p.

[25] E. Balmes, Structural Dynamics Toolbox \& FEM link, User's guide, SDTools, Paris, France, 2011.

[26] A. Mathieu, L. Maxit, N. Totaro, J.L. Guyader, Convergence acceleration using the residual shape technique when solving structure-acoustic coupling with the Patch Transfer Functions method. Computers and Structures, 88 (2010) 728-736.

[27] C.A. Felippa, Introduction to finite element methods, University of Colorado, Boulder, USA, 2001.

[28] G.-B. Warburton, The vibration of rectangular plates, Proceedings of the Institution of Mechanical Engineers 168 (1954) 371-381.

[29] J.P. Arenas, On the vibration analysis of rectangular clamped plates using the virtual work principle. Journal of Sound and Vibration, 266 (2003) 912-918.

[30] C.Q. Howard, Modal mass of clamped beams and clamped plates, Journal of Sound and Vibration, 301 (2007) 410-414. 


\section{APPENDIX A. COMPARISON OF DMF RESULTS ON THE DAVID AND MENELLE TEST CASE [20]}

The plate-water filled cavity system of Ref. [20] constitutes a reference case in the literature. The analytical and experimental results proposed in this paper are very close and are used as the reference in this appendix to validate the DMF calculations. The test case [20] is composed of a clamped rectangular plate coupled with a parallelipedic water-filled cavity. A full description of the system and a modal analysis are proposed in [20]. We recall here the geometrical and mechanical parameters considered in the DMF calculation, without comments. The plate is $170 \mathrm{~mm}$ long, $150 \mathrm{~mm}$ wide, $4 \mathrm{~mm}$ thick, clamped at its four edges and it is made of steel $\left(E=1.62 \times 10^{11} \mathrm{~Pa}, \rho=7800 \mathrm{~kg} / \mathrm{m}^{3}, v=0.3, \eta=0.008\right)$. The cavity has five rigid walls and is filled with water $\left(c_{0}=1500 \mathrm{~m} / \mathrm{s}, \rho_{0}=1000 \mathrm{~kg} / \mathrm{m}^{3}\right)$. The viscosity of the internal fluid $\tau_{F}$ is calculated with Eq. (12) in [20] from the fluid dynamic viscosity which is fixed at 0.001. A damping loss factor for the acoustic mode $q, \eta_{q}$ is then given by $\tau_{F} \omega_{q}$.

The same coordinate system as that shown in Fig. 3 is considered. The plate is excited by a normal mechanical force at point $M_{e}$ of coordinates $(0.051,0.105,0.31 \mathrm{~m})$. Two receiving points are defined: $M_{1}$ on the plate at coordinates $(0.1385,0.0625,0.31 \mathrm{~m})$, and $M_{2}$ at the bottom of the cavity at coordinates $(0.02,0.02,0.0124 \mathrm{~m})$.

Expressions for the natural frequencies, mode shapes and modal mass of the clamped plate can be found in the literature [28-30]. DMF can then be easily applied on this case. 17 plate modes below $12.5 \mathrm{kHz}$ are taken into account. Different DMF calculations including non resonant acoustic modes defined by (26) with different values of $q_{z}^{\max }$ are performed. Contrary to the case treated in Sec. 3, they show that $q_{z}^{\max }=39$ does not fully ensure the convergence of the modal decompositions. After analysis, this can be explained by the fact that the plate is thinner than that of Sec. 3 (i.e. $4 \mathrm{~mm}$ vs. $8 \mathrm{~mm}$ ). The added fluid mass compared to the plate mass per unit area is therefore larger for the present case than in Sec. 3. A value of 80 for $q_{z}^{\max }$ gives a better result and no improvements are observed for higher values. Finally, 3919 acoustic modes are considered.

The DMF results are compared in Fig. A.1 with the analytical results proposed in [20]. We recall that perfect agreement of the analytical prediction with measurements was observed in [20]. Comparison of Fig. A.1 fully validates the DMF calculations when the non resonant acoustic modes spatially coincident with the plate are taken into account. 
(a)

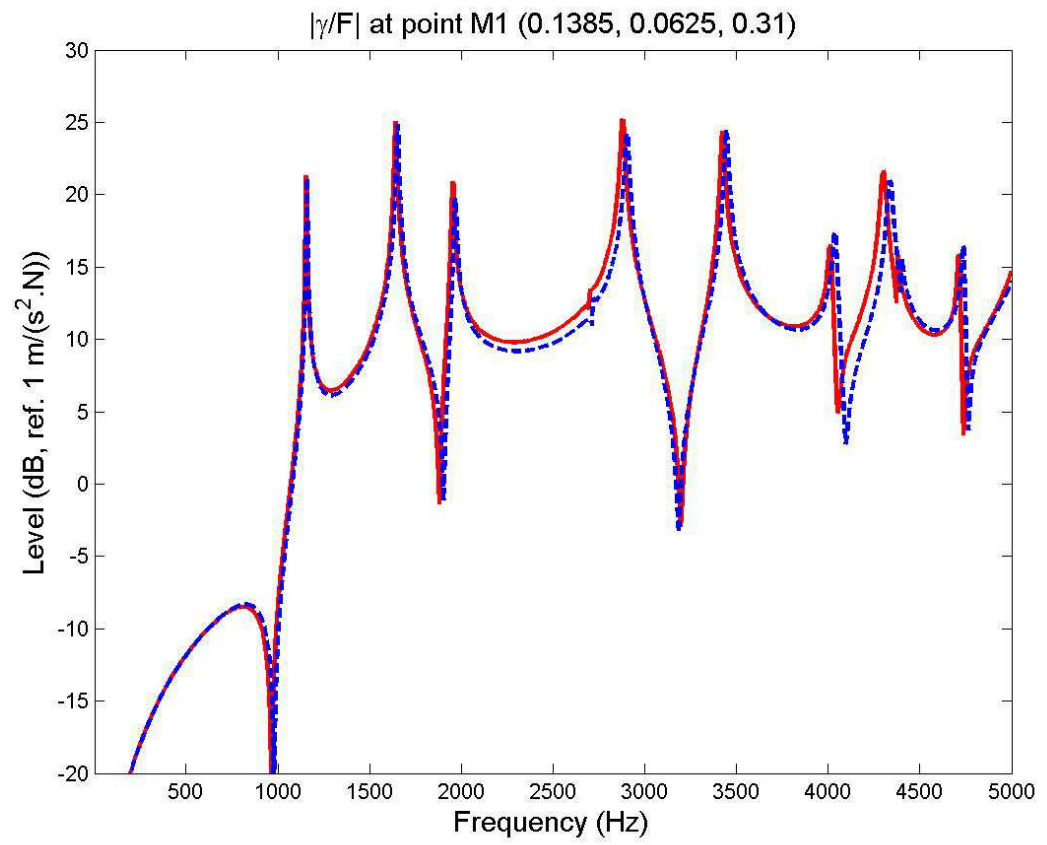

(b)

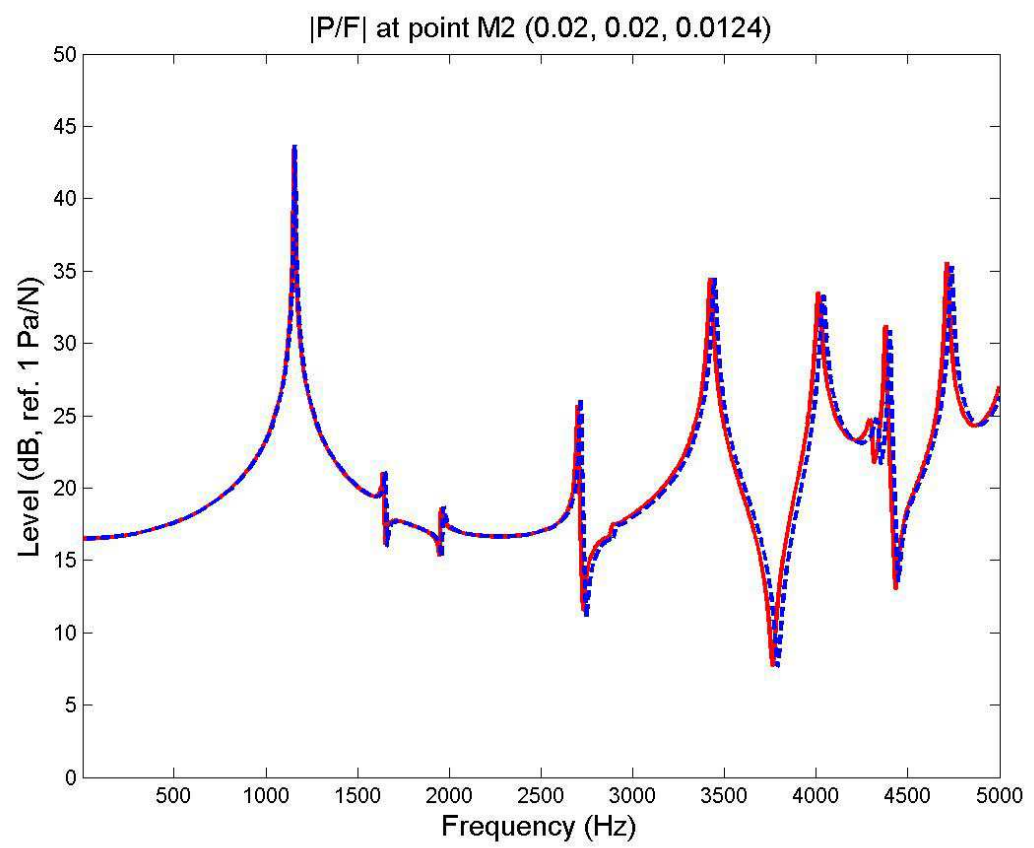

Fig. A.1. Comparison of the DMF results (dashed line) with the analytical results given in [20] (full line). (a), Acceleration at the point $M_{1}$; (b) Pressure at point $M_{2}$. 


\section{APPENDIX B. SUBSYSTEM MODES AND INTERMODAL WORKS FOR THE PLATE-CAVITY SYSTEM}

Let us consider the system described in section 3 composed of a rectangular simply-supported plate coupled with a parallelepiped cavity. The modal information used for the DMF calculation is given in this appendix for the present case.

\section{B.1 - PLATE DISPLACEMENT MODES}

A rectangular plate simply supported at its edges of dimension $L_{x} \times L_{y}$ is considered. For mode $p$ of orders $\left(p_{x}, p_{y}\right) \in[1, \infty[\times[1, \infty[$, we obtain the modal angular frequency

$$
\omega_{p}=\sqrt{\frac{D}{\rho h}}\left(\frac{p_{x} \pi x}{L_{x}}\right)^{2}+\left(\frac{p_{y} \pi x}{L_{y}}\right)^{2},
$$

the displacement mode shape,

$$
W_{p}(x, y)=\sin \left(\frac{p_{x} \pi x}{L_{x}}\right) \sin \left(\frac{p_{y} \pi y}{L_{y}}\right),
$$

and the modal mass $M_{p}\left(\right.$ defined by $\left.M_{p}=\rho h \int_{S} W_{p}^{2} d V\right)$,

$$
M_{p}=\frac{\rho h L_{x} L_{y}}{4}
$$

where $D$ is the flexural rigidity of the plate.

\section{B.2 - CAVITY PRESSURE MODES}

We consider the rectangular cavity of dimension $L_{x} \times L_{y} \times L_{z}$. The blocked modes considered in the DMF consist of the normal modes of the cavity with rigid walls. For mode $q$ of orders $\left(q_{x}, q_{y}, q_{z}\right) \in\left(\left[1, \infty[)^{3}\right.\right.$, we obtain the modal angular frequency,

$$
\omega_{q}=c_{0} \sqrt{\left(\frac{q_{x} \pi}{L_{x}}\right)^{2}+\left(\frac{q_{y} \pi}{L_{y}}\right)^{2}+\left(\frac{q_{z} \pi}{L_{z}}\right)^{2}},
$$

the pressure mode shape,

$$
p_{q}(x, y, z)=\cos \left(\frac{q_{x} \pi x}{L_{x}}\right) \cos \left(\frac{q_{y} \pi y}{L_{y}}\right) \cos \left(\frac{q_{z} \pi z}{L_{z}}\right),
$$

and the modal stiffness $K_{q}\left(\right.$ defined by $\left.K_{q}=\frac{1}{\rho_{0} c_{0}^{2}} \int_{\Omega} p_{q}^{2} d V\right)$,

$$
K_{q}=\frac{L_{x} L_{y} L_{z}}{8 \varepsilon_{q} \rho_{0} c_{0}^{2}}
$$

where $\varepsilon_{q}=\left(\frac{1}{2}\right)^{\Xi}$, and $\Xi$, the number of non-zero modal order $\left(q_{x}, q_{y}, q_{z}\right)$. 


\section{B.3 - MODAL INTERACTION WORK}

Considering the definition of the intermodal work (8) and the modes shapes (B.2, B.5), we obtain:

$$
W_{p q}=\left\{\begin{array}{l}
\frac{(-1)^{q_{z}} p_{x} L_{x} p_{y} L_{y}}{\pi^{2}}\left[\frac{(-1)^{p_{x}+q_{x}}-1}{q_{x}^{2}-p_{x}^{2}}\right]\left[\frac{(-1)^{p_{y}+q_{y}}-1}{q_{y}{ }^{2}-p_{y}{ }^{2}}\right], \text { if } p_{x} \neq q_{x}, p_{y} \neq q_{y}, \\
0, \text { otherwise. }
\end{array}\right.
$$




\section{APPENDIX C. SEA MODELLING}

In this appendix we give a description of the SEA model of the test case shown in Fig. 3.

The plate-cavity system is decomposed into two SEA subsystems: the plate is subsystem 1 and the cavity subsystem 2. For an excitation of subsystem 1 in the frequency band of central angular frequency $\omega_{c}$, the SEA equations are given by [6]:

$$
\omega_{c}\left[\begin{array}{cc}
\eta_{1}+\eta_{12} & -\eta_{21} \\
-\eta_{12} & \eta_{2}+\eta_{21}
\end{array}\right]\left[\begin{array}{c}
E_{1} \\
E_{2}
\end{array}\right]=\left[\begin{array}{c}
P_{i n j} \\
0
\end{array}\right],
$$

where: $-E_{1}, E_{2}$, are the total energy of subsystem 1 and 2, respectively;

- $\eta_{12}, \eta_{21}$ are the coupling loss factors defined below;

- $\eta_{1}, \eta_{2}$ are the damping loss factors of subsystem 1 and 2, respectively;

- $P_{i n j}$ is the power injected by the external force.

The coupling loss factor from the plate to the cavity, $\eta_{12}$, is evaluated from the radiation efficiency, $\sigma_{r}$ by

$$
\eta_{12}=\frac{\rho_{0} c_{0}}{\omega_{c} \rho_{e} h} \sigma_{r}
$$

where $\rho_{e}$ is the effective mass density of the plate

$$
\rho_{e}=\rho\left(1+\frac{\rho_{0}}{\rho h \sqrt{k_{f}^{2}-k_{0}^{2}}}\right),
$$

with $k_{0}$ and $k_{f}$ being the acoustic wavenumber of the heavy fluid and the flexural wavenumber of the plate at the angular frequency $\omega_{c}$ respectively.

The effective mass density (C.3) was obtained by considering an infinite fluid loading plate [17]. It allows taking the fluid added mass effect into account.

Below the critical frequency, (i.e. $\mu=k_{f} / k_{0}>1$ ), the radiation efficiency from Leppington's model is given by

$$
\sigma_{r}=\frac{L_{x}+L_{y}}{\pi \mu k_{0} L_{x} L_{y} \sqrt{\mu^{2}-1}}\left[\ln \left(\frac{\mu+1}{\mu-1}\right)+\frac{2 \mu}{\mu^{2}-1}\right],
$$

with $\mu=k_{f} / k_{0}$.

The coupling loss factor from the cavity to the plate, $\eta_{21}$, is obtained from the reciprocity relation,

$$
\eta_{21}=\eta_{12} n_{1} / n_{2}
$$

where $n_{1}, n_{2}$ are the modal densities of the plate and the cavity, respectively. They can be estimated from the asymptotic formula,

$$
n_{1}=\frac{L_{x} L_{y}}{4 \pi} \sqrt{\frac{\rho_{e} h}{D}} \text { and } n_{2}=\frac{L_{x} L_{y} L_{z} \omega_{c}^{2}}{\pi^{2} c_{0}^{3}} .
$$


Considering a white noise force excitation of unit ASD (i.e. $S_{F F}=1 \mathrm{~N}^{2} / \mathrm{Hz}$ ) in the frequency band of width $\Delta \omega$, the power injected by this force in the plate can be estimated by

$$
P_{i n j}=\frac{n_{1} \Delta \omega}{4 \rho_{e} h L_{x} L_{y}} .
$$

Using Eq. (C.2-C.7) for estimating the coupling loss factors and the injected power, the total energy of the plate and the cavity can be obtained by inverting the equation system (C.1). 